\title{
Differential variational-hemivariational inequalities: existence, uniqueness, stability, and convergence
}

\author{
Guo-ji Tang, Jinxia Cen, Van Thien Nguyen and Shengda Zeng®
}

\begin{abstract}
The goal of this paper is to study a comprehensive system called differential variational-hemivariational inequality which is composed of a nonlinear evolution equation and a time-dependent variational-hemivariational inequality in Banach spaces. Under the general functional framework, a generalized existence theorem for differential variational-hemivariational inequality is established by employing KKM principle, Minty's technique, theory of multivalued analysis, the properties of Clarke's subgradient. Furthermore, we explore a wellposedness result for the system, including the existence, uniqueness, and stability of the solution in mild sense. Finally, using penalty methods to the inequality, we consider a penalized problem-associated differential variational-hemivariational inequality, and examine the convergence result that the solution to the original problem can be approached, as a parameter converges to zero, by the solution of the penalized problem.
\end{abstract}

Mathematics Subject Classification. 47J20, 49J40, 58E35, 35K90, 46Txx.

Keywords. Differential variational-hemivariational inequality, KKM principle, Clarke's subgradient, well-posedness, penalty method, convergence.

\section{Introduction}

The problems called differential variational inequalities (DVIs, for short) is a kind of dynamic systems which consist of a differential equation combined

This project has received funding from the European Union's Horizon 2020 Research and Innovation Programme under the Marie Skłodowska-Curie grant agreement no. 823731CONMECH. It is supported by the National Science Center of Poland under Preludium Project No. 2017/25/N/ST1/00611, the Startup Project of Doctor Scientific Research of Yulin Normal University no. G2020ZK07, and International Project co-financed by the Ministry of Science and Higher Education of Republic of Poland under Grant No. 3792/GGPJ/H2020/2017/0. Guo-ji Tang was also supported by National Natural Science Foundation of China Grant no. 11961006, and NSF of Guangxi No. 2020GXNSFAA159100. 
with a time-dependent variational inequality. The notion of differential variational inequalities was initially introduced and systematically studied by Pang-Stewart [29] in Euclidean spaces. After that many researchers are attracted to boost the development of theory and applications of DVIs. Because DVIs are useful for the study of models involving both dynamics and constraints in the form of inequalities. They arise in many applications: electrical circuits with ideal diodes, Coulomb friction problems for contacting bodies, economical dynamics, dynamic traffic networks. The most representative results are as follows: Loi [20] applied the method of integral guiding functions to explore a multi-parameter global bifurcation theorem for differential inclusions with the periodic condition and then employed the abstract results to the study of the two-parameter global bifurcation of periodic solutions for a class of differential variational inequalities in Euclidean spaces; LiuZeng-Motreanu $[13,17,18]$ and Liu-Migórski-Zeng [16] proved the existence of solutions for a class of differential mixed variational inequalities in Banach spaces through applying the theory of semigroups, Filippov implicit function lemma and fixed point theorems for condensing multivalued operators; Chen-Wang [1] in 2014 used the idea of DVIs to investigate a dynamic Nash equilibrium problem of multiple players with shared constraints and dynamic decision processes; Nguyen-Tran [28] considered a model of infinite dimensional differential variational inequalities formulated by a parabolic differential inclusion and an elliptic variational inequality, and utilized the theory of measure of noncompactness to prove the existence of global solutions as well as global attractor for the semi-flow governed by the differential variational inequality. For more details on these topics the reader is welcome to consult $[2,6,8,10,11,14,15,21,22,24,34,35]$ and the references therein.

Recently, the concept of differential hemivariational inequalities was first proposed by Liu-Zeng-Motreanu [19]. However, a natural question why there is a need to study the differential hemivariational inequalities has been raised. More recently, the papers [23] and [37] have delivered a positive answer to this question. In the paper [23], the authors used a temporally semidiscrete method based on the backward Euler difference scheme, i.e., the Rothe method, and a feedback iterative technique to prove the existence of solutions for a class of differential hemivariational inequalities of hyperbolicparabolic type, and employed the theoretical results to a dynamic adhesive viscoelastic contact problem with friction. However, the paper [37] was devoted to adopt the idea of differential hemivariational inequalities to analyze a frictional quasistatic contact problem for viscoelastic materials with adhesion in which the friction and contact conditions are described by the Clarke's generalized gradient of nonconvex and nonsmooth functionals, and the constitutive relation is modeled by the fractional Kelvin-Voigt law.

The aim of the present paper is devoted to develop new mathematical tools and methods for differential hemivariational inequalities. Let $\left(V,\|\cdot\|_{V}\right)$ and $\left(E,\|\cdot\|_{E}\right)$ be reflexive and separable Banach spaces. Also, let $K$ be a nonempty, closed, and convex subset of $V$. In what follows, we denote by $\langle\cdot, \cdot\rangle$ the duality pairing between $V$ and its dual $V^{*}$. Assume that $A: D(A) \subset E \rightarrow$ $E$ is the infinitesimal generator of a $C_{0}$-semigroup $e^{A t}$ in $E$. Let $0<T<\infty$, 
and $\left(X,\|\cdot\|_{X}\right)$ be a separable Banach space with its dual $\left(X^{*},\|\cdot\|_{X^{*}}\right)$. Given nonlinear functions $f:[0, T] \times E \times V \rightarrow E$ and $g:[0, T] \times E \times V \rightarrow V^{*}$, a locally Lipschitz function $J: X \rightarrow \mathbb{R}$, a bounded linear operator $\gamma: V \rightarrow X$, and a bifunction $\varphi: V \times V \rightarrow \mathbb{R} \cup\{-\infty,+\infty\}$, this paper is concerned with the study of the following generalized differential variational-hemivariational inequality: find functions $x:[0, T] \rightarrow E$ and $u:[0, T] \rightarrow K$ such that

$$
\begin{cases}x^{\prime}(t)=A x(t)+f(t, x(t), u(t)) & \text { for a.e. } t \in[0, T], \\ u(t) \in S O L(K ; g(t, x(t), \cdot), J, \varphi) & \text { for a.e. } t \in[0, T], \\ x(0)=x_{0}, & \end{cases}
$$

where $S O L(K ; g(t, x(t), \cdot), J, \varphi)$ stands for the solution set of the following generalized variational-hemivariational inequality: given $t \in[0, T]$ and $x(t) \in$ $E$, find $u(t) \in K$ such that

$$
\begin{aligned}
& \langle g(t, x(t), u(t)), v-u(t)\rangle+J^{0}(\gamma u(t) ; \gamma(v-u(t))) \\
& +\varphi(v, u(t)) \geq 0 \text { for all } v \in K .
\end{aligned}
$$

From the previous work $[13,16-18]$, we now provide the definition of solutions of problem (1) in the mild sense.

Definition 1.1. A pair of functions $(x, u)$, with $x \in C([0, T] ; E)$ and $u:[0, T] \rightarrow$ $K$ integral, is called a mild solution of problem (1) if

$$
x(t)=e^{A t} x_{0}+\int_{0}^{t} e^{A(t-s)} f(s, x(s), u(s)) \mathrm{d} s
$$

for all $t \in[0, T]$, where $u(s) \in S O L(K ; g(s, x(s), \cdot), J, \varphi)$ for a.e. $s \in[0, T]$. If $(x, u)$ is a mild solution of problem $(1)$, then $x$ is called the mild trajectory and $u$ is the variational control trajectory.

The main contributions of the paper are threefold. First, using KKM principle, Minty's approach, and the properties of Clarke's subgradient, we prove that the solution set of variational-hemivariational inequality (2) is nonempty, bounded, closed, and convex. As a result, the measurability and upper semicontinuity for variational-hemivariational inequality (2) with respect to the time variable and state variable are illustrated. Second, by applying a fixed point theorem for history-dependent operators, a well-posedness result for differential variational-hemivariational inequality (1), including the existence, uniqueness, and stability of the solution in mild sense, is established. Finally, the penalty methods are employed to differential variationalhemivariational inequality (1) to consider a penalized problem, problem (20) corresponding to original problem (1) (see Sect. 4), and examine the convergence result that the solution to the original problem can be approached, as a parameter converges to zero, by the solution of the penalized problem.

The outline of the paper is as follows. Basic notation and preliminary material needed in the sequel are recalled in Sect. 2. In Sects. 3 and 4, we deliver the main results of the paper which include existence, uniqueness, stability and convergence of the solution in mild sense for differential variationalhemivariational inequality (1). Section 5 gives a conclusion of the paper . 


\section{Mathematical background}

In this section, we briefly review basic notation and some results which are needed in the sequel. For more details, we refer to monographs $[3,4,25,36]$.

Throughout the paper, we denote by $\langle\cdot, \cdot\rangle_{Y^{*} \times Y}$ the duality pairing between a Banach space $Y$ and its dual $Y^{*}$. The norm in a normed space $Y$ is denoted by $\|\cdot\|_{Y}$. Given a subset $D$ of $Y$, we write $\|D\|_{Y}=\sup \left\{\|v\|_{Y} \mid v \in Y\right\}$. If no confusion arises, we often drop the subscripts. For any nonempty set $X$, we denote by $P(X)$ the collection of its nonempty subsets. Besides, we denote by $\mathcal{L}\left(Y_{1}, Y_{2}\right)$ the space of linear and bounded operators from a normed space $Y_{1}$ to a normed space $Y_{2}$ endowed with the usual norm $\|\cdot\|_{\mathcal{L}\left(Y_{1}, Y_{2}\right)}$. In what follows, the symbols " $\rightarrow$ " and " $\rightarrow$ " denote the strong and the weak convergence in various spaces which will be specified.

Definition 2.1. Let $\left(X,\|\cdot\|_{X}\right)$ be a reflexive Banach space with its dual $X^{*}$ and $A: X \rightarrow X^{*}$. We say that

(i) $A$ is monotone, if for all $u, v \in X$, we have $\langle A u-A v, u-v\rangle \geq 0$.

(ii) $A$ is strongly monotone with constant $m_{A}>0$, if $\langle A u-A v, u-v\rangle \geq$ $m_{A}\|u-v\|_{X}^{2}$ for all $u, v \in X$.

(iii) $A$ is pseudomonotone, if $A$ is a bounded operator and for every sequence $\left\{x_{n}\right\} \subseteq X$ converging weakly to $x \in X$ such that $\lim \sup \left\langle A x_{n}, x_{n}-x\right\rangle \leq$ 0 , we have $\langle A x, x-y\rangle \leq \liminf \left\langle A x_{n}, x_{n}-y\right\rangle$ for all $y \in X$.

(iv) $A$ is hemicontinuous, if for all $u, v, w \in X$, the function $\lambda \mapsto\langle A(u+$ $\lambda v), w\rangle$ is continuous on $[0,1]$.

It is obvious that $A: X \rightarrow X^{*}$ is pseudomonotone if and only if $A$ is bounded and $x_{n} \rightarrow x$ in $X$ with $\lim \sup \left\langle A x_{n}, x_{n}-x\right\rangle \leq 0$ imply $\lim \left\langle A x_{n}, x_{n}-\right.$ $x\rangle=0$ and $A x_{n} \rightarrow A x$ in $X^{*}$. Furthermore, if $A \in \mathcal{L}\left(X, X^{*}\right)$ is nonnegative, then it is pseudomonotone.

Let $X$ be a Banach space with its dual space $X^{*}$. A function $f: X \rightarrow$ $\overline{\mathbb{R}}:=\mathbb{R} \cup\{+\infty\}$ is called proper, convex, and lower semicontinuous, if it fulfills, respectively, the following conditions:

$$
\begin{aligned}
& \mathrm{D}(f):=\{u \in X \mid f(u)<+\infty\} \neq \emptyset, \\
& f(\lambda u+(1-\lambda) v) \leq \lambda f(u)+(1-\lambda) f(v) \text { for all } \lambda \in[0,1] \text { and } u, v \in X, \\
& f(u) \leq \liminf _{n \rightarrow \infty} f\left(u_{n}\right) \text { for all sequences }\left\{u_{n}\right\} \subset X \text { with } u_{n} \rightarrow u .
\end{aligned}
$$

In the meantime, we review the definitions and properties of semicontinuous multivalued mappings.

Definition 2.2. Let $X$ and $Y$ be topological spaces, and $F: X \rightarrow P(Y)$ be a multivalued mapping. We say that $F$ is

(i) upper semicontinuous (u.s.c., for short) at $x \in X$ if, for every open set $O \subset Y$ with $F(x) \subset O$ there exists a neighborhood $N(x)$ of $x$ such that $F(N(x)):=\cup_{y \in N(x)} F(y) \subset O$. If this holds for every $x \in X$, then $F$ is called upper semicontinuous.

(ii) closed at $x_{0} \in X$, if for every sequence $\left\{\left(x_{n}, y_{n}\right)\right\} \subset \operatorname{Gr}(F)$ such that $\left(x_{n}, y_{n}\right) \rightarrow\left(x_{0}, y_{0}\right)$ in $X \times Y$, we have $\left(x_{0}, y_{0}\right) \in \operatorname{Gr}(F)$, where $\operatorname{Gr}(F)$ is 
the graph of the multivalued mapping $F$ defined by

$$
\operatorname{Gr}(F):=\{(x, y) \in X \times Y \mid y \in F(x)\} .
$$

We say that $F$ is closed (or $F$ has a closed graph), if it is closed at every $x_{0} \in X$.

The following theorem gives a criterium for upper semicontinuity.

Proposition 2.3. Let $F: X \rightarrow P(Y)$, with $X$ and $Y$ topological spaces. The statements below are equivalent:

(i) $F$ is upper semicontinuous;

(ii) for every closed set $C \subset Y$, the set $F^{-}(C)$ is closed in $X$

$$
F^{-}(C):=\{x \in X \mid F(x) \cap C \neq \emptyset\}
$$

(iii) for every open set $O \subset Y$, the set $F^{+}(O)$ is open in $X$

$$
F^{+}(O):=\{x \in X \mid F(x) \subset O\} .
$$

Definition 2.4. Let $E$ and $V$ be Banach spaces and let $I \subset \mathbb{R}$ be an interval. We say that $F: I \times E \rightarrow P(V)$ is superpositionally measurable if, for every measurable multivalued mapping $Q: I \rightarrow P(E)$ with compact values, the superposition $\Phi: I \rightarrow P(V)$ given by $\Phi(t)=F(t, Q(t))$ is measurable.

Indeed, it is quite difficult to examine if a multivalued mapping is superpositionally measurable using definition. Fortunately, the following theorem provides a necessary criterion to validate whether a multivalued mapping is superpositionally measurable.

Theorem 2.5. Let $F: I \times E \rightarrow P(V)$ be a multivalued mapping. If $t \mapsto F(t, u)$ is measurable on $I$ for all $u \in E$ and $u \mapsto F(t, u)$ is upper or lower semicontinuous for a.e. $t \in I$, then $F$ is superpositionally measurable.

Furthermore, we recall the well-known result, KKM principle, see Ky Fan [5], which will be used in Sect. 3 to verify the existence of solutions to generalized variational-hemivariational inequality (2).

Lemma 2.6. Let $K$ be a nonempty subset of a Hausdorff topological vector space $V$, and let $G: K \rightarrow P(V)$ be a multivalued mapping satisfying

(i) $G$ is a KKM mapping, namely for any $\left\{v_{1}, v_{2}, \ldots, v_{n}\right\} \subset K$, one has that its convex hull co $\left\{v_{1}, v_{2}, \ldots, v_{n}\right\}$ is contained in $\cup_{i=1}^{n} G\left(v_{i}\right)$;

(ii) $G(v)$ is closed in $V$ for every $v \in K$;

(iii) $G\left(v_{0}\right)$ is compact in $V$ for some $v_{0} \in K$.

Then it holds $\cap_{v \in K} G(v) \neq \emptyset$.

A function $J: X \rightarrow \mathbb{R}$ is called locally Lipschitz continuous at $u \in X$, if there exist a neighborhood $N(u)$ of $u$ and a constant $L_{u}>0$ such that

$$
|J(w)-J(v)| \leq L_{u}\|w-v\|_{X} \text { for all } w, v \in N(u) .
$$


Definition 2.7. Given a locally Lipschitz function $J: X \rightarrow \mathbb{R}$, we denote by $J^{0}(u ; v)$ the generalized (Clarke) directional derivative of $J$ at the point $u \in X$ in the direction $v \in X$ defined by

$$
J^{0}(u ; v)=\limsup _{\lambda \rightarrow 0^{+}, w \rightarrow u} \frac{J(w+\lambda v)-J(w)}{\lambda} .
$$

The generalized gradient of $J: X \rightarrow \mathbb{R}$ at $u \in X$ is given by

$$
\partial J(u)=\left\{\xi \in X^{*} \mid J^{0}(u ; v) \geq\langle\xi, v\rangle \text { for all } v \in X\right\} .
$$

In fact, the generalized gradient and generalized directional derivative of a locally Lipschitz function enjoy many nice properties and rich calculus. Here we just collect below some basic and critical results, see cf. [25, Proposition $3.23]$.

Proposition 2.8. Assume that $J: X \rightarrow \mathbb{R}$ is a locally Lipschitz function. Then we have

(i) for every $x \in X$, the function $X \ni v \mapsto J^{0}(x ; v) \in \mathbb{R}$ is positively homogeneous and subadditive, i.e., $J^{0}(x ; \lambda v)=\lambda J^{0}(x ; v)$ for all $\lambda \geq 0$, $v \in X$ and $J^{0}\left(x ; v_{1}+v_{2}\right) \leq J^{0}\left(x ; v_{1}\right)+J^{0}\left(x ; v_{2}\right)$ for all $v_{1}, v_{2} \in X$, respectively.

(ii) for every $v \in X$, it holds $J^{0}(x ; v)=\max \{\langle\xi, v\rangle: \xi \in \partial J(x)\}$.

(iii) the function $X \times X \ni(u, v) \mapsto J^{0}(u ; v) \in \mathbb{R}$ is upper semicontinuous.

Additionally, we recall the notion of the penalty operators, see [31].

Definition 2.9. Let $X$ be a Banach space and $K$ be a nonempty subset of $X$. An operator $P: X \rightarrow X^{*}$ is called a penalty operator of set $K$ if $P$ is bounded, demicontinuous, monotone and $K=\left\{u \in X \mid P u=0_{X^{*}}\right\}$.

Note that if $K$ is a nonempty, closed and convex subset of reflexive Banach space $X$, then the operator $P=\mathcal{J}\left(I-P_{K}\right)$ is a penalty operator of $K$, where $\mathcal{J}: X \rightarrow X^{*}$ is the duality map on $X$ defined by

$$
\mathcal{J}(x)=\left\{x^{*} \in X^{*} \mid\left\langle x^{*}, x\right\rangle=\|x\|_{X}^{2}=\left\|x^{*}\right\|_{X^{*}}^{2}\right\} \text { for } x \in X,
$$

$I$ is the identity map on $X$, and $P_{K}: X \rightarrow X$ is the projection operator of $K$ (see [4, Proposition 1.3.27]).

Definition 2.10. An operator $\mathcal{R}: C([0, T] ; X) \rightarrow C([0, T] ; X)$ is called history dependent if there exists a constant $L_{\mathcal{R}}>0$ such that

$$
\|(\mathcal{R} u)(t)-(\mathcal{R} v)(t)\|_{X} \leq L_{\mathcal{R}} \int_{0}^{t}\|u(s)-v(s)\|_{X} \mathrm{~d} s
$$

for all $u, v \in C([0, T] ; X)$ and $t \in[0, T]$.

An important property of history-dependent operators is provided by the following fixed point result, see, e.g. [32, Theorem 67].

Lemma 2.11. If $\mathcal{R}: C([0, T] ; X) \rightarrow C([0, T] ; X)$ is a history-dependent operator then there exists a unique function $u^{*} \in C([0, T] ; X)$ such that $\mathcal{R} u^{*}=u^{*}$. 


\section{A well-posedness result for differential variational-hemivariatinal inequalities}

This section is devoted to prove a well-posedness result for differential variational-hemivariational inequality, problem (1), including the existence, uniqueness, and continuous dependence of solution with respect to initial data.

We assume that the data of problem (1) read as follows.

$H(A): A: D(A) \subset E \rightarrow E$ is the infinitesimal generator of a $C_{0}$-semigroup $\overline{e^{A t}}$ in $E$ such that $\sup _{t \in[0, T]}\left\|e^{A t}\right\| \leq M_{A}$ for some $M_{A}>0$.

$H(K): K$ is a nonempty, closed, and convex subset of $V$.

$\overline{H(J)}: J: X \rightarrow \mathbb{R}$ is a locally Lipschitz function.

$\overline{H(\gamma)}: \gamma: V \rightarrow X$ is a linear, bounded, and compact operator.

$\overline{H(g)}: g:[0, T] \times E \times V \rightarrow V^{*}$ is such that

(i) $g(\cdot, \cdot, u):[0, T] \times E \rightarrow V^{*}$ is continuous for all $u \in V$;

(ii) the following inequality holds:

$$
\liminf _{\lambda \rightarrow 0^{+}}\langle g(t, x, \lambda v+(1-\lambda) u), v-u\rangle \leq\langle g(t, x, u), v-u\rangle
$$

for all $u, v \in K$ and $(t, x) \in[0, T] \times E$;

(iii) there exist a bounded function $\rho:[0, T] \times \mathbb{R}_{+} \rightarrow \mathbb{R}$, a function $r: \mathbb{R}_{+} \rightarrow \mathbb{R}$ with $r(s) \rightarrow+\infty$ as $s \rightarrow+\infty$ and an element $u_{0} \in K$ such that

$$
\begin{aligned}
& \left\langle g(t, x, v), v-u_{0}\right\rangle+\inf _{\xi \in \partial J(\gamma v)}\left\langle\xi, \gamma\left(v-u_{0}\right)\right\rangle_{X^{*} \times X} \\
& \geq r\left(\|v\|_{V}\right)\|v\|_{V}+\left(1+\|v\|_{V}\right) \rho\left(t,\|x\|_{E}\right)
\end{aligned}
$$

(iv) for each $(t, x) \in[0, T] \times E$, the multivalued mapping $u \mapsto g(t, x, u)+$ $\gamma^{*} \partial J(\gamma u)$ is monotone.

$H(\varphi)$ : The function $\varphi: V \times V \rightarrow \mathbb{R} \cup\{-\infty,+\infty\}$ is such that

(i) $v \mapsto \varphi(v, u)$ is convex for all $u \in V$;

(ii) $u \mapsto \varphi(v, u)$ is concave and upper semicontinuous for all $v \in V$;

(iii) for all $v \in K$, we have $\varphi(v, v)=0$.

$H(f)$ : The nonlinear function $f:[0, T] \times E \times V \rightarrow E$ satisfies the following conditions:

(i) for every $(t, x) \in[0, T] \times E$ and every convex set $D \subset K$, the set $f(t, x, D)$ is convex in $E$;

(ii) there exists $\psi \in L_{+}^{1}(0, T)$ such that

$\|f(t, x, u)\|_{E} \leq \psi(t)\left(1+\|x\|_{E}+\|u\|_{V}\right)$ for all $(t, x, u) \in[0, T] \times E \times K ;$

(iii) $t \mapsto f(t, x, u)$ is measurable on $[0, T]$, for every $(x, u) \in E \times V$;

(iv) $f(t, \cdot, \cdot): E \times V \rightarrow E$ is continuous for a.e. $t \in[0, T]$;

(v) there exists $k \in L_{+}^{1}(0, T)$ such that

$$
\left\|f\left(t, x_{0}, u\right)-f\left(t, x_{1}, u\right)\right\|_{E} \leq k(t)\left\|x_{0}-x_{1}\right\|_{E}
$$

for $x_{0}, x_{1} \in E$, all $u \in K$ and a.e. $t \in[0, T]$. 
To establish the existence result for problem (1), first, we will exploit the following generalized elliptic variational-hemivariational inequality: find $u \in K$ such that

$$
\langle Q(u), v-u\rangle+J^{0}(\gamma u ; \gamma(v-u))+\varphi(v, u) \geq 0 \text { for all } v \in K,
$$

where $Q: K \rightarrow V^{*}$ is a given mapping. For simplicity, in what follows, denote by $S O L(K ; Q, J, \varphi)$ the solution set of problem $(3)$.

Theorem 3.1. Assume that $H(J), H(\varphi), H(\gamma), H(K)$ and the following conditions are satisfied

(i) $Q: K \rightarrow V^{*}$ is monotone such that

$$
\liminf _{\lambda \rightarrow 0^{+}}\langle Q(\lambda v+(1-\lambda) u), v-u\rangle \leq\langle Q(u), v-u\rangle \quad \text { for all } u, v \in K
$$

(ii) if the set $K$ is unbounded in $V$, there exists $u_{0} \in K$ such that

$$
\liminf _{v \in K,\|v\|_{V} \rightarrow \infty} \frac{\left\langle Q(v), v-u_{0}\right\rangle+\inf _{\xi \in \partial J(\gamma v)}\left\langle\xi, \gamma\left(v-u_{0}\right)\right\rangle_{X * \times X}}{\|v\|_{V}}=+\infty .
$$

Then the solution set of problem $(3), \operatorname{SOL}(K ; Q, J, \varphi)$, is nonempty, bounded and weakly closed in $V$.

Proof. We first show that the solution set of problem $(3), \operatorname{SOL}(K ; Q, J, \varphi)$, is nonempty. To do so, we shall consider two situations that $K$ is bounded and $K$ is unbounded.

Suppose that $K$ is bounded in $V$. Consider a multivalued mapping $G: K \rightarrow P(K)$ defined by

$G(v):=\left\{u \in K:\langle Q(v), v-u\rangle+J^{0}(\gamma u ; \gamma(v-u))+\varphi(v, u) \geq 0\right\} \quad$ for all $v \in K$.

Obviously, for each $v \in K$, the set $G(v)$ is nonempty, owing to $v \in G(v)$ for each $v \in K$. Besides it asserts that $G$ has weakly closed values. Let $\left\{u_{n}\right\} \subset G(v)$ be a weakly convergent sequence, namely $u_{n} \rightarrow u$ as $n \rightarrow \infty$ for some $u \in V$. Hence, for each $n$, one has

$$
\left\langle Q(v), v-u_{n}\right\rangle+J^{0}\left(\gamma u_{n} ; \gamma\left(v-u_{n}\right)\right)+\varphi\left(v, u_{n}\right) \geq 0 .
$$

Notice that $K$ is closed and convex, so it has $u \in K$. On the other side, the compactness of $\gamma$, hypothesis $H(\varphi)$ (ii) and the fact $(u, v) \mapsto J^{0}(u ; v)$ is upper semicontinuous reveal that

$\limsup _{n \rightarrow \infty} J^{0}\left(\gamma u_{n} ; \gamma\left(v-u_{n}\right)\right) \leq J^{0}(\gamma u ; \gamma(v-u))$ and $\limsup _{n \rightarrow \infty} \varphi\left(v, u_{n}\right) \leq \varphi(v, u)$.

Passing to the upper limit as $n \rightarrow \infty$ in inequality (4) and taking into account the above inequalities, it finds

$$
\begin{aligned}
& \langle Q(v), v-u\rangle+J^{0}(\gamma u ; \gamma(v-u))+\varphi(v, u) \\
& \quad \geq \limsup _{n \rightarrow \infty}\left\langle Q(v), v-u_{n}\right\rangle+\limsup _{n \rightarrow \infty} J^{0}\left(\gamma u_{n} ; \gamma\left(v-u_{n}\right)\right)+\limsup _{n \rightarrow \infty} \varphi\left(v, u_{n}\right) \\
& \quad \geq \limsup _{n \rightarrow \infty}\left[\left\langle Q(v), v-u_{n}\right\rangle+J^{0}\left(\gamma u_{n} ; \gamma\left(v-u_{n}\right)\right)+\varphi\left(v, u_{n}\right)\right] \\
& \quad \geq 0 ;
\end{aligned}
$$

thus is $u \in G(v)$. Therefore, $G(v)$ is weakly closed for each $v \in K$. 
Multifunction $G$ is a KKM mapping. Indeed, arguing by contradiction, there exist a finite subset $\left\{v_{1}, v_{2}, \ldots, v_{N}\right\} \subset K$ and $u_{0}=\sum_{i=1}^{N} t_{i} v_{i}$ with $t_{i} \in[0,1]$ and $\sum_{i=1}^{N} t_{i}=1$ such that

$$
u_{0} \notin \bigcup_{i=1}^{N} G\left(v_{i}\right) .
$$

This means that

$$
\left\langle Q\left(v_{i}\right), v_{i}-u_{0}\right\rangle+\varphi\left(v_{i}, u_{0}\right)+J^{0}\left(\gamma u_{0} ; \gamma v_{i}-\gamma u_{0}\right)<0 .
$$

The monotonicity of $Q$ ensures that

$$
\left\langle Q\left(u_{0}\right), v_{i}-u_{0}\right\rangle+\varphi\left(v_{i}, u_{0}\right)+J^{0}\left(\gamma u_{0} ; \gamma v_{i}-\gamma u_{0}\right)<0
$$

for each $i=1, \ldots, N$. Employing the convexity of $v \mapsto \varphi(v, u)$, as well as the positive homogeneity and subadditivity of $v \mapsto J^{0}(u ; v)$, we obtain

$$
\begin{aligned}
0 & =\left\langle Q\left(u_{0}\right), u_{0}-u_{0}\right\rangle+\varphi\left(u_{0}, u_{0}\right)+J^{0}\left(\gamma u_{0} ; \gamma u_{0}-\gamma u_{0}\right) \\
& =\left\langle Q\left(u_{0}\right), \sum_{i=1}^{N} t_{i} v_{i}-u_{0}\right\rangle+\varphi\left(\sum_{i=1}^{N} t_{i} v_{i}, u_{0}\right)+J^{0}\left(\gamma u_{0} ; \gamma \sum_{i=1}^{N} t_{i} v_{i}-\gamma u_{0}\right) \\
& \leq \sum_{i=1}^{N} t_{i}\left[\left\langle Q\left(u_{0}\right), v_{i}-u_{0}\right\rangle+\varphi\left(v_{i}, u_{0}\right)+J^{0}\left(\gamma u_{0} ; \gamma v_{i}-\gamma u_{0}\right)\right] \\
& <0 .
\end{aligned}
$$

This generates a contradiction, so, we conclude that $G$ is a KKM mapping.

Keeping in mind that $K$ is bounded, closed and convex, it follows from reflexivity of $V$ that $K$ is also weakly compact. This implies that $G(v)$ is weakly compact too, for every $v \in K$, since $G$ has weakly closed values. By invoking KKM principle, Lemma 2.6, with respect to the weak topology of $V$, it has

$$
\bigcap_{v \in K} G(v) \neq \emptyset
$$

Hence, we can find an element $u^{*} \in K$ such that

$$
\left\langle Q(v), v-u^{*}\right\rangle+J^{0}\left(\gamma u^{*} ; \gamma\left(v-u^{*}\right)\right)+\varphi\left(v, u^{*}\right) \geq 0
$$

for all $v \in K$. For any $w \in K$ and $t \in(0,1)$, we take $v=v_{t}:=t w+(1-t) u^{*}$ into the above inequality to get

$$
\left\langle Q\left(v_{t}\right), w-u^{*}\right\rangle+J^{0}\left(\gamma u^{*} ; \gamma\left(w-u^{*}\right)\right)+\varphi\left(w, u^{*}\right) \geq 0,
$$

where we have utilized the condition $H(\varphi)$ (iii). Passing to the lower limit as $t \rightarrow 0^{+}$in the above inequality and using condition (i), one obtains

$$
\left\langle Q\left(u^{*}\right), w-u^{*}\right\rangle+J^{0}\left(\gamma u^{*} ; \gamma\left(w-u^{*}\right)\right)+\varphi\left(w, u^{*}\right) \geq 0 .
$$

Since $w \in K$ is arbitrary, this signifies that $u^{*} \in K$ is a solution to problem (3).

Furthermore, we consider the situation that $K$ is unbounded. For each $n \in \mathbb{N}$, define $K_{n} \subset K$ by

$$
K_{n}:=\left\{x \in K:\left\|x-u_{0}\right\|_{V} \leq n\right\},
$$


where $u_{0}$ is given in the hypothesis (ii). So, for each $n \in \mathbb{N}$, we are able to find a solution $u_{n} \in K_{n}$ to the following problem:

$$
\left\langle Q\left(u_{n}\right), v-u_{n}\right\rangle+J^{0}\left(\gamma u_{n} ; \gamma\left(v-u_{n}\right)\right)+\varphi\left(v, u_{n}\right) \geq 0
$$

for all $v \in K_{n}$. We affirm that there exists an integer $N_{0} \geq 1$ such that

$$
\left\|u_{N_{0}}-u_{0}\right\|_{V}<N_{0} .
$$

If it does not hold, then for each $n \in \mathbb{N}$, the equality $\left\|u_{n}-u_{0}\right\|_{V}=n$ is true. This points out $\left\|u_{n}\right\|_{V} \rightarrow \infty$ as $n \rightarrow \infty$. Putting $v=u_{0}$ into (5), it turns out

$$
\left\langle Q\left(u_{n}\right), u_{0}-u_{n}\right\rangle+J^{0}\left(\gamma u_{n} ; \gamma\left(u_{0}-u_{n}\right)\right)+\varphi\left(u_{0}, u_{n}\right) \geq 0 .
$$

However, Proposition 2.8(ii) says that there is an element $\xi_{n} \in \partial J\left(\gamma u_{n}\right)$ such that

$$
J^{0}\left(\gamma u_{n} ; \gamma\left(u_{0}-u_{n}\right)\right)=\left\langle\xi_{n}, \gamma\left(u_{0}-u_{n}\right)\right\rangle_{X^{*} \times X} .
$$

Observe that $u \mapsto-\varphi(v, u)$ is convex, see assumption $H(\varphi)$ (ii), from [33, Proposition 1.29], we have

$$
-\varphi\left(u_{0}, v\right) \geq c_{\varphi}+\left\langle\eta_{\varphi}, v\right\rangle
$$

for all $v \in V$, where $c_{\varphi} \in \mathbb{R}$ and $\eta_{\varphi} \in V^{*}$ only depend on $u_{0}$. Combining with (7)-(9), it yields

$$
\left\langle Q\left(u_{n}\right), u_{n}-u_{0}\right\rangle+\inf _{\xi \in \partial J\left(\gamma u_{n}\right)}\left\langle\xi, \gamma\left(u_{n}-u_{0}\right)\right\rangle_{X^{*} \times X}-\left|c_{\varphi}\right|-\left\|\eta_{\varphi}\right\|_{V^{*}}\left\|u_{n}\right\|_{V} \leq 0 .
$$

Remembering that $\left\|u_{n}\right\|_{V} \rightarrow \infty$ as $n \rightarrow \infty$ and condition (ii), we imply

$$
\begin{aligned}
+\infty & =\liminf _{n \rightarrow \infty} \frac{\left\langle Q\left(u_{n}\right), u_{n}-u_{0}\right\rangle+\inf _{\xi \in \partial J\left(\gamma u_{n}\right)}\left\langle\xi, \gamma\left(u_{n}-u_{0}\right)\right\rangle_{X^{*} \times X}}{\left\|u_{n}\right\|_{V}} \\
& \leq\left\|\eta_{\varphi}\right\|_{V^{*}}+\liminf _{n \rightarrow \infty} \frac{\left|c_{\varphi}\right|}{\left\|u_{n}\right\|_{V}} \\
& =\left\|\eta_{\varphi}\right\|_{V^{*}} .
\end{aligned}
$$

This leads a contradiction. Hence, the claim in (6) is valid.

Let $w \in K$ be arbitrary. Assume that $N_{0} \in \mathbb{N}$ and $u_{N_{0}}$ are such that (6) holds. It allows us to pick a sufficiently small $t>0$ satisfying

$$
(1-t) u_{N_{0}}+t w \in K_{N_{0}} .
$$

Inserting $v=(1-t) u_{N_{0}}+t w$ into (5) for $n=N_{0}$, it reads

$$
\left\langle Q\left(u_{N_{0}}\right), w-u_{N_{0}}\right\rangle+J^{0}\left(\gamma u_{N_{0}} ; \gamma\left(w-u_{N_{0}}\right)\right)+\varphi\left(w, u_{N_{0}}\right) \geq 0
$$

for all $w \in K$. This concludes that $u_{N_{0}}$ is a solution to problem (3).

Next, we show that $\operatorname{SOL}(K ; Q, J, \varphi)$ is weakly closed in $V$. Let $\left\{u_{n}\right\} \subset$ $\operatorname{SOL}(K ; Q, J, \varphi)$ be a weakly convergent sequence, i.e., $u_{n} \rightarrow u$ in $V$ for some $u \in V$. Then we have

$$
\left\langle Q\left(u_{n}\right), v-u_{n}\right\rangle+J^{0}\left(\gamma u_{n} ; \gamma\left(v-u_{n}\right)\right)+\varphi\left(v, u_{n}\right) \geq 0 \text { for all } v \in K .
$$

The monotonicity of $Q$ suggests

$$
\left\langle Q(v), v-u_{n}\right\rangle+J^{0}\left(\gamma u_{n} ; \gamma\left(v-u_{n}\right)\right)+\varphi\left(v, u_{n}\right) \geq 0 \text { for all } v \in K .
$$


The upper semicontinuity of $w \mapsto \varphi(v, w)$ and $(w, v) \mapsto J^{0}(w ; v)$ combined with the compactness of $\gamma$ and hypothesis $H(\varphi)$ (ii) indicates

$$
\limsup _{n \rightarrow \infty} J^{0}\left(\gamma u_{n} ; \gamma\left(v-u_{n}\right)\right) \leq J^{0}(\gamma u ; \gamma(v-u)) \text { and } \limsup _{n \rightarrow \infty} \varphi\left(v, u_{n}\right) \leq \varphi(v, u) .
$$

Passing to the upper limit in inequality (10) and taking into account the above inequalities, we have

$$
\begin{aligned}
& \langle Q(v), v-u\rangle+J^{0}(\gamma u ; \gamma(v-u))+\varphi(v, u) \\
& \quad \geq \limsup _{n \rightarrow \infty}\left\langle Q(v), v-u_{n}\right\rangle+\limsup _{n \rightarrow \infty} J^{0}\left(\gamma u_{n} ; \gamma\left(v-u_{n}\right)\right)+\limsup _{n \rightarrow \infty} \varphi\left(v, u_{n}\right) \\
& \geq \limsup _{n \rightarrow \infty}\left[\left\langle Q(v), v-u_{n}\right\rangle+J^{0}\left(\gamma u_{n} ; \gamma\left(v-u_{n}\right)\right)+\varphi\left(v, u_{n}\right)\right] \\
& \geq 0 \text { for all } v \in K .
\end{aligned}
$$

Now, we use the Minty approach again to obtain $u \in S O L(K ; Q, J, \varphi)$. Therefore, $\operatorname{SOL}(K ; Q, J, \varphi)$ is weakly closed in $V$.

It is enough to demonstrate that $\operatorname{SOL}(K ; Q, J, \varphi)$ is bounded. Suppose that $\operatorname{SOL}(K ; Q, J, \varphi)$ is unbounded. Therefore, we can find a sequence $\left\{u_{n}\right\} \subset$ $\operatorname{SOL}(K ; Q, J, \varphi)$ such that $\left\|u_{n}\right\|_{V} \rightarrow+\infty$ as $n \rightarrow \infty$. As before we did, a simple calculation gives

$$
+\infty=\liminf _{n \rightarrow \infty} \frac{\left\langle Q\left(u_{n}\right), u_{n}-u_{0}\right\rangle+\inf _{\xi \in \partial J\left(\gamma u_{n}\right)}\left\langle\xi, \gamma\left(u_{n}-u_{0}\right)\right\rangle_{X^{*} \times X}}{\left\|u_{n}\right\|_{V}} \leq\left\|\eta_{\varphi}\right\|_{V^{*}}
$$

This reaches a contradiction. Consequently, we conclude that $\operatorname{SOL}(K ; Q, J, \varphi)$ is bounded.

Additionally, we have the following two corollaries.

Corollary 3.2. Assume that $H(J), H(\gamma), H(K)$, and conditions (i)-(ii) of Theorem 3.1 are fulfilled. If $\phi: K \rightarrow \overline{\mathbb{R}}:=\mathbb{R} \cup\{+\infty\}$ is a proper, convex and lower semicontinuous function, then the solution set of the following variational-hemivariational inequality

$$
\begin{aligned}
& \text { find } u \in K \text { such that }\langle Q(u), v-u\rangle+J^{0}(\gamma u ; \gamma(v-u)) \\
& \quad+\phi(v)-\phi(u) \geq 0 \text { for all } v \in K
\end{aligned}
$$

is nonempty, bounded, and weakly closed.

Proof. It is easy to verify that the function $\varphi: V \times V \rightarrow \mathbb{R} \cup\{-\infty,+\infty\}$ defined by $\varphi(v, u)=\phi(v)-\phi(u)$ reads hypothesis $H(\varphi)$. The conclusion of the corollary is a direct consequence of Theorem 3.1.

Corollary 3.3. Under the assumptions $H(\varphi), H(K)$, if conditions (i)-(ii) of Theorem 3.1 hold with $J \equiv 0$, then the solution set of the following generalized variational inequality

find $u \in K$ such that $\langle Q(u), v-u\rangle+\varphi(v, u) \geq 0$ for all $v \in K$, is nonempty, bounded, and weakly closed.

For more particular cases of Theorem 3.1, Corollaries 3.2 and 3.3, the reader is welcome to refer $[12,13,18,27]$.

The following lemma delivers a convexity result for set $\operatorname{SOL}(K ; Q, J, \varphi)$. 
Lemma 3.4. Suppose that $Q: K \rightarrow V^{*}$ is such that

$$
\liminf _{\lambda \rightarrow 0^{+}}\langle Q(\lambda v+(1-\lambda) u), v-u\rangle \leq\langle Q(u), v-u\rangle \quad \text { for all } u, v \in K,
$$

the multivalued mapping $u \mapsto Q u+\gamma^{*} \partial J(\gamma u)$ is monotone, and $v \mapsto \varphi(v, u)$ is convex for all $u \in K$ and $u \mapsto \varphi(v, u)$ is concave for all $v \in K$ with $\varphi(u, u)=0$ for all $u \in K$. Then the set $\operatorname{SOL}(K ; Q, J, \varphi)$ is convex, when it is nonempty.

Proof. Assume that $\operatorname{SOL}(K ; Q, J, \varphi)$ is nonempty. Let $u_{1}, u_{2} \in S O L$ $(K ; Q, J, \varphi)$ and $t \in(0,1)$ be arbitrary. Then for $i=1,2$ we have

$$
\left\langle Q\left(u_{i}\right), v-u_{i}\right\rangle+J^{0}\left(\gamma u_{i} ; \gamma\left(v-u_{i}\right)\right)+\varphi\left(v, u_{i}\right) \geq 0
$$

for all $v \in K$. Using the property, Proposition 2.8(ii), we are able to find an element $\xi_{i} \in \partial J\left(\gamma u_{i}\right)$ such that

$$
J^{0}\left(\gamma u_{i} ; \gamma\left(v-u_{i}\right)\right)=\left\langle\xi_{i}, \gamma\left(v-u_{i}\right)\right\rangle_{X^{*} \times X}
$$

for $i=1,2$. Taking into account (11) and monotonicity of $u \mapsto Q u+$ $\gamma^{*} \partial J(\gamma u)$, it yields

$$
\begin{aligned}
0 & \leq\left\langle Q\left(u_{i}\right), v-u_{i}\right\rangle+J^{0}\left(\gamma u_{i} ; \gamma\left(v-u_{i}\right)\right)+\varphi\left(v, u_{i}\right) \\
& =\left\langle Q\left(u_{i}\right)+\gamma^{*} \xi_{i}, v-u_{i}\right\rangle+\varphi\left(v, u_{i}\right) \\
& \leq\left\langle Q(v)+\gamma^{*} \xi_{v}, v-u_{i}\right\rangle+\varphi\left(v, u_{i}\right)
\end{aligned}
$$

for all $\xi_{v} \in \partial J(\gamma v)$ and all $v \in K, i=1,2$. Denote $u_{t}=t u_{1}+(1-t) u_{2}$. The above inequalities and the concavity of $u \mapsto \varphi(v, u)$ point out

$$
\begin{aligned}
& \left\langle Q(v)+\gamma^{*} \xi_{v}, v-u_{t}\right\rangle+\varphi\left(v, u_{t}\right) \\
& \geq t\left[\left\langle Q(v)+\gamma^{*} \xi_{v}, v-u_{1}\right\rangle+\varphi\left(v, u_{1}\right)\right]+(1-t)[\langle Q(v) \\
& \left.\left.\quad+\gamma^{*} \xi_{v}, v-u_{2}\right\rangle+\varphi\left(v, u_{2}\right)\right] \\
& \geq 0
\end{aligned}
$$

for all $\xi_{v} \in \partial J(\gamma v)$ and all $v \in K$. For any $w \in K$, inserting $v_{\lambda}=\lambda w+(1-$ ג) $u_{t}$ into (12) implies

$$
\begin{aligned}
& \lambda\left\langle Q\left(v_{\lambda}\right)+\gamma^{*} \xi_{v_{\lambda}}, w-u_{t}\right\rangle \\
& \quad+\lambda \varphi\left(w, u_{t}\right)+(1-\lambda) \varphi\left(u_{t}, u_{t}\right) \geq 0 \text { for all } \xi_{v_{\lambda}} \in \partial J\left(\gamma v_{\lambda}\right),
\end{aligned}
$$

thus is,

$$
\begin{aligned}
0 & \leq\left\langle Q\left(v_{\lambda}\right)+\gamma^{*} \xi_{v_{\lambda}}, w-u_{t}\right\rangle+\varphi\left(w, u_{t}\right) \\
& \leq\left\langle Q\left(v_{\lambda}\right), w-u_{t}\right\rangle+J^{0}\left(\gamma v_{\lambda} ; \gamma\left(w-u_{t}\right)\right)+\varphi\left(w, u_{t}\right) .
\end{aligned}
$$

Passing to the lower limit as $\lambda \rightarrow 0$ in the above inequality and using condition (i) of Theorem 3.1, it emerges

$$
\left\langle Q\left(u_{t}\right), w-u_{t}\right\rangle+J^{0}\left(\gamma u_{t} ; \gamma\left(w-u_{t}\right)\right)+\varphi\left(w, u_{t}\right) \geq 0,
$$

here we have applied the upper semicontinuity of $(u, v) \mapsto J^{0}(u ; v)$, see Proposition 2.8(iii), and the fact, $\liminf \left[f\left(x_{n}\right)+g\left(x_{n}\right)\right] \leq \liminf f\left(x_{n}\right)+$ $\lim \sup g\left(x_{n}\right)$. Since $w \in K$ is arbitrary, so, we conclude that $u_{t} \in S O L$ $(K ; Q, J, \varphi)$, namely the set $\operatorname{SOL}(K ; Q, J, \varphi)$ is convex. 
As a byproduct of the proof of Lemma 3.4, we also provide a Minty type equivalence result for problem (3).

Lemma 3.5. Under the assumptions of Lemma 3.4, $u \in \operatorname{SOL}(K ; Q, J, \varphi)$ if and only if $u$ solves the following Minty variational-hemivariational inequality: find $u \in K$ such that

$$
\langle Q(v), v-u\rangle+J^{0}(\gamma v ; \gamma(v-u))+\varphi(v, u) \geq 0
$$

for all $v \in K$.

Combining Theorem 3.1 with Lemma 3.4, we obtain the following theorem.

Theorem 3.6. Suppose that the condition (ii) of Theorem $3.1, H(J), H(\varphi)$, $H(\gamma)$ and $H(K)$ are fulfilled. If, in addition, $Q: K \rightarrow V^{*}$ is such that

$$
\liminf _{\lambda \rightarrow 0^{+}}\langle Q(\lambda v+(1-\lambda) u), v-u\rangle \leq\langle Q(u), v-u\rangle \quad \text { for all } u, v \in K,
$$

and the multivalued mapping $u \mapsto Q u+\gamma^{*} \partial J(\gamma u)$ is monotone, then the solution set of problem (3), $\operatorname{SOL}(K ; Q, J, \varphi)$, is nonempty, bounded, closed and convex in $V$.

Consider a multivalued mapping $U:[0, T] \times E \rightarrow P(K)$ defined by

$$
\begin{aligned}
U(t, x):= & \left\{u \in K \mid\langle g(t, x, u), v-u\rangle+J^{0}(\gamma u ; \gamma(v-u))\right. \\
& +\varphi(v, u) \geq 0 \text { for all } v \in K\}
\end{aligned}
$$

for all $(t, x) \in[0, T] \times E$. Moreover, the following theorem reveals that $U$ is well defined, strongly-weakly upper semicontinuous, and superpositionally measurable.

Theorem 3.7. Assume hypotheses $H(g), H(J), H(\varphi), H(\gamma)$ and $H(K)$ are satisfied. Then multivalued mapping $U:[0, T] \times E \rightarrow P(K)$ given in (13) is well defined and satisfies the following properties:

$\left(U_{1}\right) U$ is strongly-weakly upper semicontinuous;

$\left(U_{2}\right) U$ is superpositionally measurable in the sense of Definition 2.4.

Proof. In fact, Theorem 3.6 implies that for each $(t, x) \in[0, T] \times E$ the set $U(t, x)$ is nonempty, bounded, closed, and convex. So, the mapping $U:[0, T] \times$ $E \rightarrow P(K)$ is well defined.

We now apply Proposition 2.3 to verify the assertion $\left(U_{1}\right)$. It reminds us to demonstrate that for each weakly closed subset $C \subset K$, the set

$$
U^{-}(C):=\{(t, x) \in[0, T] \times E \mid U(t, x) \cap C \neq \emptyset\}
$$

is closed in $\mathbb{R} \times E$. Let $\left\{\left(t_{n}, x_{n}\right)\right\} \subset U^{-}(C)$ be a sequence such that $\left(t_{n}, x_{n}\right) \rightarrow$ $(t, x)$ in $\mathbb{R} \times E$ as $n \rightarrow \infty$. Thus, there exists a sequence $\left\{u_{n}\right\} \subset K$ with $u_{n} \in U\left(t_{n}, x_{n}\right) \cap C$ for each $n \in \mathbb{N}$, especially, $\left\langle g\left(t_{n}, x_{n}, u_{n}\right), v-u_{n}\right\rangle+J^{0}\left(\gamma u_{n} ; \gamma\left(v-u_{n}\right)\right)+\varphi\left(v, u_{n}\right) \geq 0$ for all $v \in K$

and all $n \in \mathbb{N}$. We prove that the sequence $\left\{u_{n}\right\}$ is uniformly bounded. Arguing by contradiction, passing to a subsequence if necessary, we may say 
that $\left\|u_{n}\right\|_{V} \rightarrow \infty$ as $n \rightarrow \infty$. Taking $v=u_{0}$ into (14) and using hypothesis $H(g)($ iii) imply

$$
\begin{aligned}
0 & \geq\left\langle g\left(t_{n}, x_{n}, u_{n}\right)+\gamma^{*} \xi_{u_{n}}, u_{n}-u_{0}\right\rangle-\varphi\left(u_{0}, u_{n}\right) \\
& \geq r\left(\left\|u_{n}\right\|_{V}\right)\left\|u_{n}\right\|_{V}+\left(1+\left\|u_{n}\right\|_{V}\right) \rho\left(t_{n},\left\|x_{n}\right\|_{E}\right)-\varphi\left(u_{0}, u_{n}\right) \\
& \geq r\left(\left\|u_{n}\right\|_{V}\right)\left\|u_{n}\right\|_{V}+\left(1+\left\|u_{n}\right\|_{V}\right) \rho\left(t_{n},\left\|x_{n}\right\|_{E}\right)-\left|c_{\varphi}\right|-\left\|\eta_{\varphi}\right\| V^{*}\left\|u_{n}\right\|_{V}
\end{aligned}
$$

for some $c_{\varphi} \in \mathbb{R}$ and $\eta_{\varphi} \in V^{*}$. Then we have

$$
r\left(\left\|u_{n}\right\|_{V}\right)+\frac{1+\left\|u_{n}\right\|_{V}}{\left\|u_{n}\right\|_{V}} \rho\left(t_{n},\left\|x_{n}\right\|_{E}\right)-\frac{\left|c_{\varphi}\right|}{\left\|u_{n}\right\|_{V}}-\left\|\eta_{\varphi}\right\|_{V^{*}} \leq 0 .
$$

Recall that $\rho$ is a bounded function, $\left\{\left(t_{n}, x_{n}\right)\right\}$ is bounded in $[0, T] \times E$, and $r(s) \rightarrow+\infty$ as $s \rightarrow+\infty$, it takes the lower limit as $n \rightarrow \infty$ in the above inequality to get

$$
\begin{aligned}
+\infty= & \liminf _{n \rightarrow \infty}\left(r\left(\left\|u_{n}\right\|_{V}\right)+\frac{1+\left\|u_{n}\right\|_{V}}{\left\|u_{n}\right\|_{V}} \rho\left(t_{n},\left\|x_{n}\right\|_{E}\right)\right) \\
& -\liminf _{n \rightarrow \infty} \frac{\left|c_{\varphi}\right|}{\left\|u_{n}\right\|_{V}}-\left\|\eta_{\varphi}\right\|_{V^{*}} \leq 0 .
\end{aligned}
$$

This generates a contradiction, hence, $\left\{u_{n}\right\}$ is uniformly bounded. Without loss of generality, we may assume that $u_{n} \rightarrow u$ in $V$ as $n \rightarrow \infty$, for some $u \in K$.

On the other hand, Lemma 3.5 and (14) guarantee

$$
\left\langle g\left(t_{n}, x_{n}, v\right), v-u_{n}\right\rangle+J^{0}\left(\gamma v ; \gamma\left(v-u_{n}\right)\right)+\varphi\left(v, u_{n}\right) \geq 0 \text { for all } v \in K
$$

and all $n \in \mathbb{N}$. Remember that $u \mapsto \varphi(v, u)$ is concave and upper semicontinuous. Passing to the upper limit as $n \rightarrow \infty$ in (15), it yields

$$
\begin{aligned}
0 & \leq \limsup _{n \rightarrow \infty}\left[\left\langle g\left(t_{n}, x_{n}, v\right), v-u_{n}\right\rangle+J^{0}\left(\gamma v ; \gamma\left(v-u_{n}\right)\right)+\varphi\left(v, u_{n}\right)\right] \\
& \leq \limsup _{n \rightarrow \infty}\left\langle g\left(t_{n}, x_{n}, v\right), v-u_{n}\right\rangle+\limsup _{n \rightarrow \infty} J^{0}\left(\gamma v ; \gamma\left(v-u_{n}\right)\right)+\limsup _{n \rightarrow \infty} \varphi\left(v, u_{n}\right) \\
& \leq\langle g(t, x, v), v-u\rangle+J^{0}(\gamma v ; \gamma(v-u))+\varphi(v, u)
\end{aligned}
$$

for all $v \in K$. We have used the facts, $(t, x) \mapsto g(t, x, v)$ is continuous, $\gamma$ is compact, $u \mapsto \varphi(v, u)$ is weakly upper semicontinuous (because it is concave and upper semicontinuous). Employing Lemma 3.5 again, we conclude that $u \in U(t, x)$. The latter coupled with the weak closedness of $C$ implies $u \in U(t, x) \cap C$, i.e., $(t, x) \in U^{-}(C)$. Therefore, $U$ is strongly-weakly upper semicontinuous.

Concerning the proof of $\left(U_{2}\right)$, Proposition 6.2 .4 of [30] points out that if, for all $x \in E$ and $v \in V$, the function $t \mapsto d(v, U(t, x))$ is measurable, then $U(\cdot, x)$ is measurable as well. In fact, if for each $\lambda \geq 0$ the set $M_{\lambda}:=\{t \in$ $[0, T] \mid d(v, U(t, x)) \leq \lambda\}$ is measurable, then the function $t \mapsto d(v, U(t, x))$ is measurable too. Moreover, here, we will show that for each $\lambda \geq 0$ the set $M_{\lambda}:=\{t \in[0, T] \mid d(v, U(t, x)) \leq \lambda\}$ is closed, so, it is measurable. Notice that $U$ has closed and convex values, hence, for every $n \in \mathbb{N}$, we are able to take a unique element $u_{n} \in U\left(t_{n}, x\right)$ such that $\left\|v-u_{n}\right\|_{V}=d\left(v, U\left(t_{n}, x\right)\right) \leq \lambda$. 
As before we have done, it is not difficult to see that the sequence $\left\{u_{n}\right\}$ is bounded. This allows us to suppose that $u_{n} \rightarrow u$, as $n \rightarrow \infty$, for some $u \in K$. Whereas the strongly-weakly upper semicontinuity of $U$ ensures $u \in U(t, x)$. This infers

$$
d(v, U(t, x)) \leq\|v-u\|_{V} \leq \liminf _{n \rightarrow \infty}\left\|v-u_{n}\right\|_{V} \leq \lambda .
$$

This indicates that $M_{\lambda}$ is closed, so, $U(\cdot, x)$ is measurable. Consequently, from assertion $\left(U_{1}\right)$ and Theorem 2.5, we conclude the desired result $\left(U_{2}\right)$.

Invoking the same arguments with the proof of [18, Lemma 4.2 and Theorem 4.4], [13, Lemma 3.6] and Theorem 3.6, we are now in a position to conclude the following existence result to problem (1).

Theorem 3.8. Assume that $H(A), H(g), H(f), H(J), H(\gamma), H(\varphi)$ and $H(K)$ hold. Then the solution set of problem (1) in the sense of Definition 1.1 is nonempty, and the set of all mild trajectories $x$ of problem (1) is compact in $C([0, T] ; E)$.

Moreover, we shall examine a well-posedness result for problem (1). To do so, we need the following assumptions:

$\underline{H(J)^{\prime}}: J: X \rightarrow \mathbb{R}$ is a locally Lipschitz function and enjoys the following properties

(i) there exist constants $\alpha_{J} \geq 0$ and $b_{J}>0$ such that

$$
\|\partial J(w)\|_{X^{*}} \leq \alpha_{J}+b_{J}\|w\|_{X} \text { for all } w \in X ;
$$

(ii) there exists a constant $m_{J} \geq 0$ such that

$\langle\xi-\eta, w-v\rangle_{X^{*} \times X} \geq-m_{J}\|w-v\|_{X}^{2} \quad$ for all $w, v \in X$ and all $\xi \in \partial J(w), \eta \in \partial J(v)$. $\underline{H(g)^{\prime}}: g:[0, T] \times E \times V \rightarrow V^{*}$ is such that

(i) for all $(t, x) \in[0, T] \times E$, the mapping $u \mapsto g(t, x, u)$ is hemicontinuous and is uniformly strongly monotone, i.e., there exists a constant $m_{g}>0$ such that the following inequality holds

$$
\langle g(t, x, u)-g(t, x, v), u-v\rangle \geq m_{g}\|u-v\|_{V}^{2} \quad \text { for all } u, v \in V
$$

and all $(t, x) \in[0, T] \times E$;

(ii) there exists a constant $L_{g}>0$ such that

$$
\left\|g\left(t_{1}, x_{1}, u\right)-g\left(t_{2}, x_{2}, u\right)\right\|_{V^{*}} \leq L_{g}\left(\left|t_{1}-t_{2}\right|+\left\|x_{1}-x_{2}\right\|_{E}\right)
$$

for all $t_{1}, t_{2} \in[0, T], x_{1}, x_{2} \in E$ and all $u \in K$.

$\underline{H(f)^{\prime}}$ : The nonlinear function $f:[0, T] \times E \times V \rightarrow E$ satisfies the following conditions:

(i) for all $(x, u) \in E \times V$, the function $t \mapsto f(t, x, u)$ is measurable on $[0, T]$;

(ii) the function $t \mapsto f\left(t, 0_{E}, 0_{V}\right)$ belongs to $L^{1}([0, T] ; E)$;

(iii) there exists a function $\psi \in L_{+}^{\infty}(0, T)$ such that

$$
\left\|f\left(t, x_{1}, u_{1}\right)-f\left(t, x_{2}, u_{2}\right)\right\|_{E} \leq \psi(t)\left(\left\|x_{1}-x_{2}\right\|_{E}+\left\|u_{1}-u_{2}\right\|_{V}\right)
$$

for a.e. $t \in[0, T]$ and all $\left(x_{1}, u_{1}\right),\left(x_{2}, u_{2}\right) \in E \times V$. 
$\underline{H(0):} m_{g}>m_{J}\|\gamma\|_{\mathcal{L}(V, X)}^{2}$.

Remark 3.9. Assumption $H(J)^{\prime}($ ii) is usually called relaxed monotone condition (see, e.g. [25]) for the locally Lipschitz function $J$. It is equivalent to the inequality

$$
J^{0}(u ; v-u)+J^{0}(v ; u-v) \leq m_{J}\|u-v\|_{X}^{2}
$$

for all $u, v \in X$.

Theorem 3.10. Assume that $H(A), H(g)^{\prime}, H(J)^{\prime}, H(\varphi)$ with $\varphi(u, v)+\varphi(v, u)$ $\leq 0$ for all $u, v \in K, H(\gamma), H(f)^{\prime}, H(K)$, and $H(0)$ are fulfilled. Then we have

(i) for each initial point $x_{0} \in E$, differential variational-hemivariational inequality (1) possesses a unique solution $(x, u) \in C([0, T] ; E) \times C([0, T] ; K)$.

(ii) the map $x_{0} \mapsto(x, u)\left(x_{0}\right): E \rightarrow C([0, T] ; E) \times C([0, T] ; K)$ is Lipschitz continuous.

Proof. (i) For any $u_{0} \in K$ fixed, it follows from hypotheses $H(g)^{\prime}$ and $H(J)^{\prime}$ that

$$
\begin{aligned}
&\left\langle g(t, x, u), u-u_{0}\right\rangle+\left\langle\xi, \gamma\left(u-u_{0}\right)\right\rangle_{X^{*} \times X} \\
&=\left\langle g(t, x, u)-g\left(t, x, u_{0}\right), u-u_{0}\right\rangle+\langle\xi \\
&\left.-\xi_{0}, \gamma\left(u-u_{0}\right)\right\rangle_{X^{*} \times X}+\left\langle g\left(t, x, u_{0}\right)+\gamma^{*} \xi_{0}, u-u_{0}\right\rangle \\
& \geq\left(m_{g}-m_{J}\|\gamma\|_{\mathcal{L}(V, X)}^{2}\right)\left\|u-u_{0}\right\|_{V}^{2} \\
&-\left(\|\gamma\|_{\mathcal{L}(V, X)}\left\|\xi_{0}\right\|_{X^{*}}+\left\|g\left(t, x, u_{0}\right)\right\|_{V^{*}}\right)\left\|u-u_{0}\right\|_{V} \\
& \geq\left(m_{g}-m_{J}\|\gamma\|_{\mathcal{L}(V, X)}^{2}\right)\left\|u-u_{0}\right\|_{V}^{2} \\
&-\left(\|\gamma\|_{\mathcal{L}(V, X)} \alpha_{J}+\left\|g\left(t, x, u_{0}\right)-g\left(0,0_{E}, u_{0}\right)\right\|_{V^{*}}\right)\left\|u-u_{0}\right\|_{V} \\
&-\left\|g\left(0,0_{E}, u_{0}\right)\right\|_{V^{*}}\left\|u-u_{0}\right\|_{V} \\
& \geq\left(m_{g}-m_{J}\|\gamma\|_{\mathcal{L}(V, X)}^{2}\right)\left\|u-u_{0}\right\|_{V}^{2} \\
&-\left(\|\gamma\|_{\mathcal{L}(V, X)} \alpha_{J}+L_{g}\left(|t|+\|x\|_{E}\right)\right)\left\|u-u_{0}\right\|_{V} \\
&-\left\|g\left(0,0_{E}, u_{0}\right)\right\|_{V^{*}}\left\|u-u_{0}\right\|_{V}
\end{aligned}
$$

for all $\xi \in \partial J(\gamma u)$ and all $\xi_{0} \in \partial J\left(\gamma 0_{V}\right)$. Hence, $H(0)$ indicates that

$$
\begin{aligned}
\langle g(t, & \left.x, u), u-u_{0}\right\rangle+\inf _{\xi \in \partial J(\gamma u)}\left\langle\xi, \gamma\left(u-u_{0}\right)\right\rangle_{X^{*} \times X} \\
\geq & \left(m_{g}-m_{J}\|\gamma\|_{\mathcal{L}(V, X)}^{2}\right)\|u\|_{V}^{2} \\
& -\left(\|\gamma\|_{\mathcal{L}(V, X)} \alpha_{J}+L_{g}\left(|t|+\|x\|_{E}\right)+\left\|g\left(0,0_{E}, u_{0}\right)\right\|_{V^{*}}\right)\|u\|_{V} \\
& -\left(\|\gamma\|_{\mathcal{L}(V, X)} \alpha_{J}+L_{g}\left(|t|+\|x\|_{E}\right)+\left\|g\left(0,0_{E}, u_{0}\right)\right\|_{V^{*}}\right)\left\|u_{0}\right\|_{V} \\
& -\left(m_{g}-m_{J}\|\gamma\|_{\mathcal{L}(V, X)}^{2}\right)\left\|u_{0}\right\|_{V}^{2} \\
& -2\left\|u_{0}\right\|_{V}\left(m_{g}-m_{J}\|\gamma\|_{\mathcal{L}(V, X)}^{2}\right)\|u\|_{V} \\
\geq & r\left(\|u\|_{V}\right)\|u\|_{V}+\left(1+\|u\|_{V}\right) \rho\left(t,\|x\|_{E}\right)
\end{aligned}
$$


where $r: \mathbb{R}_{+} \rightarrow \mathbb{R}$ and $\rho:[0, T] \times \mathbb{R}_{+} \rightarrow \mathbb{R}$ are, respectively, defined by

$$
\begin{aligned}
r(s)= & \left(m_{g}-m_{J}\|\gamma\|_{\mathcal{L}(V, X)}^{2}\right) s \\
& -\|\gamma\|_{\mathcal{L}(V, X)} \alpha_{J}-\left\|g\left(0,0_{E}, u_{0}\right)\right\|_{V^{*}} \\
& -2\left\|u_{0}\right\|_{V}\left(m_{g}-m_{J}\|\gamma\|_{\mathcal{L}(V, X)}^{2}\right)
\end{aligned}
$$

and

$$
\begin{aligned}
\rho(t, s)= & -\left(\|\gamma\|_{\mathcal{L}(V, X)} \alpha_{J}+L_{g}(t+s)\right. \\
& \left.+\left\|g\left(0,0_{E}, u_{0}\right)\right\|_{V^{*}}\right)\left\|u_{0}\right\|_{V} \\
& -\left(m_{g}-m_{J}\|\gamma\|_{\mathcal{L}(V, X)}^{2}\right)\left\|u_{0}\right\|_{V}^{2} .
\end{aligned}
$$

Let $x \in C([0, T] ; E)$ be fixed. We now consider the following timedependent variational-hemivariational inequality: find $u:[0, T] \rightarrow K$ such that

$\langle g(t, x(t), u(t)), v-u(t)\rangle+J^{0}(\gamma u(t) ; \gamma(v-u(t)))+\varphi(v, u(t)) \geq 0 \quad$ for all $v \in K$

and all $t \in[0, T]$. We now claim that for $t \in[0, T]$ fixed inequality (17) has a unique solution $u(t)$. Indeed, under the assumptions $H(g)^{\prime}, H(J)^{\prime}, H(\varphi)$, $H(K), H(0)$ and inequality (16), we can verify all conditions of Theorem 3.6. This permits us to use Theorem 3.6 to find an element $u(t) \in K$ such that inequality (17) holds. Let $u(t)$ and $\widetilde{u}(t)$ be two solutions to problem (17). Then one has

$$
\begin{aligned}
& \langle g(t, x(t), u(t)), v-u(t)\rangle+J^{0}(\gamma u(t) ; \gamma(v-u(t))) \\
& \quad+\varphi(v, u(t)) \geq 0 \quad \text { for all } v \in K
\end{aligned}
$$

and

$\langle g(t, x(t), \widetilde{u}(t)), v-\widetilde{u}(t)\rangle+J^{0}(\gamma \widetilde{u}(t) ; \gamma(v-\widetilde{u}(t)))+\varphi(v, \widetilde{u}(t)) \geq 0 \quad$ for all $v \in K$.

Inserting $v=\widetilde{u}(t)$ into the above first inequality and $v=u(t)$ into the second one, we sum the resulting inequalities to get

$$
\begin{aligned}
& \langle g(t, x(t), u(t))-g(t, x(t), \widetilde{u}(t)), u(t)-\widetilde{u}(t)\rangle-\left(J^{0}(\gamma u(t) ; \gamma(\widetilde{u}(t)-u(t)))\right. \\
& \left.\quad+J^{0}(\gamma \widetilde{u}(t) ; \gamma(u(t)-\widetilde{u}(t)))\right) \leq \varphi(u(t), \widetilde{u}(t))+\varphi(\widetilde{u}(t), u(t)) .
\end{aligned}
$$

The latter coupled with hypotheses $H(g)^{\prime}(\mathrm{i}), H(J)^{\prime}(\mathrm{ii})$, and the fact $\varphi(w, v)+$ $\varphi(v, w) \leq 0$ for all $w, v \in K$, implies

$$
\left(m_{g}-m_{J}\|\gamma\|_{\mathcal{L}(V, X)}^{2}\right)\|u(t)-\widetilde{u}(t)\|_{V}^{2} \leq 0 .
$$

However, $H(0)$ ensures $u(t)=\widetilde{u}(t)$, namely for $t \in[0, T]$ fixed inequality (17) has a unique solution $u(t)$.

Therefore, we can find a unique function $u:[0, T] \rightarrow K$ to solve problem (17) on $[0, T]$. Further, it asserts that $u$ is a continuous function, namely $u \in C([0, T] ; K)$. For any $t_{1}, t_{2} \in[0, T]$, it has 
$\left\langle g\left(t_{i}, x\left(t_{i}\right), u\left(t_{i}\right), v-u\left(t_{i}\right)\right\rangle+J^{0}\left(\gamma u\left(t_{i}\right) ; \gamma\left(v-u\left(t_{i}\right)\right)\right)+\varphi\left(v, u\left(t_{i}\right)\right) \geq 0\right.$ for all $v \in K$ and $i=1,2$. Taking $v=u\left(t_{2}\right)$ and $v=u\left(t_{1}\right)$ into the above inequalities for $i=1$ and $i=2$, respectively, a simple calculation finds

$$
\begin{aligned}
& \left\langle g\left(t_{1}, x\left(t_{1}\right), u\left(t_{1}\right)\right)-g\left(t_{2}, x\left(t_{2}\right), u\left(t_{2}\right)\right), u\left(t_{1}\right)-u\left(t_{2}\right)\right\rangle \\
& \quad-\left(J^{0}\left(\gamma u\left(t_{1}\right) ; \gamma\left(u\left(t_{2}\right)-u\left(t_{1}\right)\right)\right)\right. \\
& \quad+J^{0}\left(\gamma u\left(t_{2}\right) ; \gamma\left(u\left(t_{1}\right)-u\left(t_{2}\right)\right)\right) \leq \varphi\left(u\left(t_{1}\right), u\left(t_{2}\right)\right)+\varphi\left(u\left(t_{2}\right), u\left(t_{1}\right)\right) \leq 0 .
\end{aligned}
$$

Then we have

$$
\begin{aligned}
& \left(m_{g}-m_{J}\|\gamma\|_{\mathcal{L}(V ; X)}^{2}\right)\left\|u\left(t_{1}\right)-u\left(t_{2}\right)\right\|_{V}^{2} \\
& \quad \leq\left\langle g\left(t_{2}, x\left(t_{2}\right), u\left(t_{2}\right)\right)-g\left(t_{1}, x\left(t_{1}\right), u\left(t_{2}\right)\right), u\left(t_{1}\right)-u\left(t_{2}\right)\right\rangle \\
& \quad \leq\left\|g\left(t_{2}, x\left(t_{2}\right), u\left(t_{2}\right)\right)-g\left(t_{1}, x\left(t_{1}\right), u\left(t_{2}\right)\right)\right\|_{V^{*}}\left\|u\left(t_{1}\right)-u\left(t_{2}\right)\right\|_{V} \\
& \quad \leq L_{g}\left(\left|t_{1}-t_{2}\right|+\left\|x\left(t_{1}\right)-x\left(t_{2}\right)\right\|_{E}\right)\left\|u\left(t_{1}\right)-u\left(t_{2}\right)\right\|_{V},
\end{aligned}
$$

which implies that

$$
\left\|u\left(t_{1}\right)-u\left(t_{2}\right)\right\|_{V} \leq \frac{L_{g}}{m_{g}-m_{J}\|\gamma\|_{\mathcal{L}(V ; X)}^{2}}\left(\left|t_{1}-t_{2}\right|+\left\|x\left(t_{1}\right)-x\left(t_{2}\right)\right\|_{E}\right) .
$$

This means $u \in C([0, T] ; K)$, thanks to $x \in C([0, T] ; E)$.

For $i=1,2$, let $x_{i} \in C([0, T] ; E)$ and $u_{i} \in C([0, T] ; K)$ be the unique solution of problem (17), namely,

$\left\langle g\left(t, x_{i}(t), u_{i}(t)\right), v-u_{i}(t)\right\rangle+J^{0}\left(\gamma u_{i}(t) ; \gamma\left(v-u_{i}(t)\right)\right)+\varphi\left(v, u_{i}(t)\right) \geq 0 \quad$ for all $v \in K$ for all $t \in[0, T]$. Putting $v=u_{2}(t)$ and $v=u_{1}(t)$ into the above inequalities for $i=1,2$, accordingly, a easy verification gives

$$
\left\|u_{1}(t)-u_{2}(t)\right\|_{V} \leq \frac{L_{g}}{m_{g}-m_{J}\|\gamma\|_{\mathcal{L}(V ; X)}^{2}}\left\|x_{1}(t)-x_{2}(t)\right\|_{E}
$$

for all $t \in[0, T]$.

For $u \in C([0, T] ; K)$ fixed, we introduce the following Cauchy problem:

$$
\left\{\begin{array}{l}
x^{\prime}(t)=A x(t)+f(t, x(t), u(t)) \quad \text { for a.e. } t \in[0, T], \\
x(0)=x_{0} .
\end{array}\right.
$$

It is clear from [9, Proposition 5.3, p.66] and [18, Section 4] that problem (19) admits a unique mild solution $x \in C([0, T] ; E)$ such that

$$
x(t)=e^{A t} x_{0}+\int_{0}^{t} e^{A(t-s)} f(s, x(s), u(s)) \mathrm{d} s \quad \text { for all } t \in[0, T] .
$$

Define a function $S: C([0, T] ; E) \rightarrow C([0, T] ; E)$ by

$$
S(x)(t)=e^{A t} x_{0}+\int_{0}^{t} e^{A(t-s)} f\left(s, x(s), u_{x}(s)\right) \mathrm{d} s
$$

for all $t \in[0, T]$, where $u_{x} \in C([0, T] ; K)$ is the unique solution to problem (17) corresponding to $x$. It is obvious that $x \in C([0, T] ; E)$ is a fixed point of $S$, if and only if $\left(x, u_{x}\right)$ is a solution to differential variational-hemivariational inequality (1). 
Next, we will show that $S$ has a unique fixed point in $C([0, T] ; E)$. For any $x_{1}, x_{2} \in C([0, T] ; E)$, it has

$$
\begin{aligned}
& \left\|S\left(x_{1}\right)(t)-S\left(x_{2}\right)(t)\right\|_{E} \\
& \quad \leq \int_{0}^{t}\left\|e^{A(t-s)}\right\|\left\|f\left(s, x_{1}(s), u_{x_{1}}(s)\right)-f\left(s, x_{2}(s), u_{x_{2}}(s)\right)\right\|_{E} \mathrm{~d} s \\
& \quad \leq M_{A} \int_{0}^{t} \psi(s)\left(\left\|x_{1}(s)-x_{2}(s)\right\|_{E}+\left\|u_{x_{1}}(s)-u_{x_{2}}(s)\right\|_{V}\right) \mathrm{d} s
\end{aligned}
$$

for all $t \in[0, T]$. This combined with (18) derives

$$
\begin{aligned}
& \left\|S\left(x_{1}\right)(t)-S\left(x_{2}\right)(t)\right\|_{E} \\
& \quad \leq M_{A} \int_{0}^{t} \psi(s)\left(1+\frac{L_{g}}{m_{g}-m_{J}\|\gamma\|_{\mathcal{L}(V, X)}^{2}}\right)\left\|x_{1}(s)-x_{2}(s)\right\|_{E} \mathrm{~d} s
\end{aligned}
$$

for all $t \in[0, T]$; hence,

$\left\|S\left(x_{1}\right)(t)-S\left(x_{2}\right)(t)\right\|_{E} \leq M_{A} c_{\psi}\left(1+\frac{L_{g}}{m_{g}-m_{J}\|\gamma\|_{\mathcal{L}(V, X)}^{2}}\right) \int_{0}^{t}\left\|x_{1}(s)-x_{2}(s)\right\|_{E} \mathrm{~d} s$ for all $t \in[0, T]$, where $c_{\psi}>0$ is such that $\psi(t) \leq c_{\psi}$ for a.e. $t \in[0, T]$. This means that $S$ is a history-dependent operator. Therefore, we are now in a position to invoke the fixed point principle, Lemma 2.11, that $S$ has a unique fixed point $x \in C([0, T] ; E)$. So, differential variational-hemivariational inequality (1) admits a unique solution $(x, u) \in C([0, T] ; E) \times C([0, T] ; K)$.

(ii) Let $x_{0}^{1}$ and $x_{0}^{2}$ be two initial points in $E$. Assertion (i) allows us to find two unique solutions $\left(x_{1}, u_{1}\right)$ and $\left(x_{2}, u_{2}\right)$ to problem (1) associated with initial points $x_{0}^{1}$ and $x_{0}^{2}$, respectively. Hence, it has

$$
\begin{aligned}
& x_{1}(t)=e^{A t} x_{0}^{1}+\int_{0}^{t} e^{A(t-s)} f\left(s, x_{1}(s), u_{1}(s)\right) \mathrm{d} s, \\
& x_{2}(t)=e^{A t} x_{0}^{2}+\int_{0}^{t} e^{A(t-s)} f\left(s, x_{2}(s), u_{2}(s)\right) \mathrm{d} s
\end{aligned}
$$

for all $t \in[0, T]$. Subtracting the above equalities, it emerges

$$
\begin{aligned}
&\left\|x_{1}(t)-x_{2}(t)\right\|_{E} \\
& \leq\left.M_{A} \int_{0}^{t} \| f\left(s, x_{1}(s), u_{1}(s)\right)-f\left(s, x_{2}(s), u_{2}(s)\right)\right) \|_{E} \mathrm{~d} s \\
& \quad+M_{A}\left\|x_{0}^{1}-x_{0}^{2}\right\|_{E} \\
& \leq M_{A}\left\|x_{0}^{1}-x_{0}^{2}\right\|_{E}+M_{A} \int_{0}^{t} \psi(s)\left(\left\|x_{1}(s)-x_{2}(s)\right\|_{E}\right. \\
& \quad\left.+\left\|u_{1}(s)-u_{2}(s)\right\|_{V}\right) \mathrm{d} s
\end{aligned}
$$

for all $t \in[0, T]$. This coupled with inequality (18) finds

$$
\begin{aligned}
& \left\|x_{1}(t)-x_{2}(t)\right\|_{E} \leq M_{A}\left\|x_{0}^{1}-x_{0}^{2}\right\|_{E} \\
& \quad+M_{A} \int_{0}^{t} \psi(s)\left(1+\frac{L_{g}}{m_{g}-m_{J}\|\gamma\|_{\mathcal{L}(V, X)}^{2}}\right)\left\|x_{1}(s)-x_{2}(s)\right\|_{E} \mathrm{~d} s
\end{aligned}
$$


for all $t \in[0, T]$. By applying Gronwall's inequality, we can see

$$
\begin{aligned}
& \max _{t \in[0, T]}\left\|x_{1}(t)-x_{2}(t)\right\|_{E} \\
& \quad \leq M_{A}\left\|x_{0}^{1}-x_{0}^{2}\right\|_{E} \exp \left(M_{A} \int_{0}^{T} \psi(s)\left(1+\frac{L_{g}}{m_{g}-m_{J}\|\gamma\|_{\mathcal{L}(V, X)}^{2}}\right) \mathrm{d} s\right) \\
& \quad \leq M_{A} \exp \left(M_{A}\left(1+\frac{L_{g}}{m_{g}-m_{J}\|\gamma\|_{\mathcal{L}(V, X)}^{2}}\right)\|\psi\|_{L^{1}[0, T]}\right)\left\|x_{0}^{1}-x_{0}^{2}\right\|_{E} .
\end{aligned}
$$

Moreover, we put the above estimate to (18) to obtain

$$
\begin{aligned}
& \max _{t \in[0, T]}\left\|u_{1}(t)-u_{2}(t)\right\|_{V} \\
& \leq \frac{L_{g} M_{A}}{m_{g}-m_{J}\|\gamma\|_{\mathcal{L}(V ; X)}^{2}} \exp \left(M_{A}\left(1+\frac{L_{g}}{m_{g}-m_{J}\|\gamma\|_{\mathcal{L}(V, X)}^{2}}\right)\|\psi\|_{L^{1}[0, T]}\right)\left\|x_{0}^{1}-x_{0}^{2}\right\|_{E} .
\end{aligned}
$$

To conclude, we can know that the map $x_{0} \mapsto(x, u)\left(x_{0}\right): E \rightarrow C([0, T] ; E) \times$ $C([0, T] ; V)$ is Lipschitz continuous with the Lipschitz constant $L>0$,

$$
L=\left(1+\frac{L_{g}}{m_{g}}\right) M_{A} \exp \left(M_{A}\left(1+\frac{L_{g}}{m_{g}-m_{J}\|\gamma\|_{\mathcal{L}(V, X)}^{2}}\right)\|\psi\|_{L^{1}[0, T]}\right),
$$

which completes the proof.

Remark 3.11. Let $\Omega \subset \mathbb{R}^{n}$ be a bounded domain with Lipschitz boundary, $E=L^{2}(\Omega)$. Indeed, the assumptions presented in the section are quite mild. To understand them deeply, we now provide the following particular examples:

(i) the linear operator $A: H_{0}^{2}(\Omega) \subset L^{2}(\Omega) \rightarrow L^{2}(\Omega), A x=\Delta x$ for all $x \in H_{0}^{2}(\Omega)$, is the generator of a $C_{0}$-semigroup $\left\{e^{A t}\right\}_{t \geq 0}$ of contractions on $L^{2}(\Omega)$.

(ii) the embedding operator $\gamma: H^{1}(\Omega) \rightarrow L^{2}(\Omega)$ is linear, bounded and compact.

(iii) $e:[0, T] \times \Omega \times \mathbb{R} \rightarrow \mathbb{R}$ and $h:[0, T] \times \Omega \times \mathbb{R} \rightarrow \mathbb{R}$ are two continuous functions such that

$$
\begin{aligned}
& \left|e\left(t, y, s_{1}\right)-e\left(t, y, s_{2}\right)\right| \leq L_{e}\left|s_{1}-s_{2}\right|, \\
& \quad\left|h\left(t, y, s_{1}\right)-h\left(t, y, s_{2}\right)\right| \leq L_{h}\left|s_{1}-s_{2}\right|
\end{aligned}
$$

for all $t \in[0, T], y \in \Omega, s_{1}, s_{2} \in \mathbb{R}$, with some $L_{e}, L_{h}>0$, then $f(t, x, u)(y):=e(t, y, x(y))+h(t, y, u(y))$ satisfies condition $H(f)^{\prime}$.

(iv) the function $j: \Omega \times \mathbb{R} \rightarrow \mathbb{R}$ is measurable such that $s \mapsto j(y, s)$ is locally Lipschitz for a.e. $y \in \Omega$ and

$$
\begin{aligned}
& |\partial j(y, s)| \leq c_{j}(1+|s|), \\
& \quad j^{0}\left(y, s_{1} ; s_{2}-s_{1}\right)+j^{0}\left(y, s_{2} ; s_{1}-s_{2}\right) \leq m_{j}\left|s_{1}-s_{2}\right|^{2}
\end{aligned}
$$

for all $y \in \Omega$, all $s, s_{1}, s_{2} \in \mathbb{R}$, with some $c_{j}>0$ and $m_{j} \geq 0$, then the function $J: L^{2}(\Omega) \rightarrow \mathbb{R}, J(u)=\int_{\Omega} j(x, u(x)) \mathrm{d} x$ for all $u \in L^{2}(\Omega)$, satisfies hypothesis $H(J)^{\prime}$. 
(v) the function $p:[0, T] \times \Omega \times \mathbb{R} \rightarrow \mathbb{R}$ is a continuous function such that $\left|p\left(t, y, s_{1}\right)-p\left(t, y, s_{2}\right)\right| \leq L_{p}\left|s_{1}-s_{2}\right|$ for all $t \in[0, T], s_{1}, s_{2} \in \mathbb{R}$ and a.e. $y \in \Omega$ with some $L_{p}>0$, then the function $g:[0, T] \times L^{2}(\Omega) \times H^{1}(\Omega) \rightarrow H^{1}(\Omega)^{*}$ defined by

$$
\langle g(t, x, u), v\rangle:=\int_{\Omega}(\nabla u(y), \nabla v(y))_{\mathbb{R}^{n}} \mathrm{~d} y+\int_{\Omega} p(t, y, x(y)) v(y) \mathrm{d} y
$$

for all $t \in[0, T], x \in L^{2}(\Omega)$ and $u, v \in H^{1}(\Omega)$, satisfies the condition $H(g)^{\prime}$.

\section{Penalty method for differential variational-hemivariational inequalities}

Penalty method as a useful tool has been widely used to the study of various optimization problems with constraints, such as Nash equilibrium problems, optimal control problems with state and input constraints of nonlinear systems, and convection-diffusion problems with characteristic layers. Recently, penalty methods for variational inequalities and hemivariational inequalities have been investigated by many authors, for numerical purposes and for proofs of solution existence, see, e.g. [7,26]. However, until now, there are no results concerning penalty methods for generalized differential variational-hemivariational inequality (1). To fill this gap, therefore, this section is devoted to provide a theoretical analysis of penalty methods for differential variational-hemivariational inequality (1), see Theorem 4.1. More precisely, we introduce a penalized problem corresponding to problem (1), and prove that the penalized problem has a unique solution $\left(x_{\rho}, u_{\rho}\right) \in C([0, T] ; E) \times C([0, T] ; V)$. Then a convergence result, the solution of original differential variational-hemivariational inequality (1) can be approximated by the penalized problem (20), see below, as the penalty parameter $\rho$ tends to zero, is established.

Let $\rho>0$ and $P: V \rightarrow V^{*}$ be a penalty operator of constraint set $K$, see Definition 2.9. The penalized problem associated with differential variational-hemivariational inequality (1) is to find functions $x_{\rho}:[0, T] \rightarrow E$ and $u_{\rho}:[0, T] \rightarrow V$ such that

$$
\left\{\begin{array}{l}
x_{\rho}^{\prime}(t)=A x_{\rho}(t)+f\left(t, x_{\rho}(t), u_{\rho}(t)\right) \quad \text { for a.e. } t \in[0, T], \\
u_{\rho}(t) \in V \text { satisfying } \\
\left\langle g\left(t, x_{\rho}(t), u_{\rho}(t)\right), v-u_{\rho}(t)\right\rangle+\frac{1}{\rho}\left\langle P u_{\rho}(t), v-u_{\rho}(t)\right\rangle+J^{0}\left(\gamma u_{\rho}(t) ; \gamma\left(v-u_{\rho}(t)\right)\right) \\
+\varphi\left(v, u_{\rho}(t)\right) \geq 0 \text { for all } v \in V \text { and for all } t \in[0, T], \\
x_{\rho}(0)=x_{0} .
\end{array}\right.
$$

The main results of the section on existence, uniqueness and convergence for problem (20) is the following.

Theorem 4.1. Assume that $H(A), H(g)^{\prime}, H(J)^{\prime}, H(\varphi)$ with $\varphi(u, v)+\varphi(v, u) \leq$ 0 for all $u, v \in K, H(\gamma), H(0), H(K)$ and $H(f)^{\prime}$ are fulfilled, and $P: V \rightarrow V^{*}$ is a penalty operator of $K$. Then we have 
(i) for each $\rho>0$, there exists a unique solution $\left(x_{\rho}, u_{\rho}\right) \in C([0, T] ; E) \times$ $C([0, T] ; V)$ to problem $(20)$.

(ii) $\left(x_{\rho}, u_{\rho}\right)$ converges to the unique solution $(x, u)$ of problem (1) as $\rho \rightarrow 0$, in the following sense:

$$
\left(x_{\rho}(t), u_{\rho}(t)\right) \rightarrow(x(t), u(t)) \text { as } \rho \rightarrow 0,
$$

for all $t \in[0, T]$.

Proof. (i) For each $\rho>0$, consider a function $g_{\rho}:[0, T] \times E \times V \rightarrow V^{*}$ defined by

$$
g_{\rho}(t, x, u)=g(t, x, u)+\frac{1}{\rho} P u \text {. }
$$

It is not difficult to corroborate that $g_{\rho}$ reads hypothesis $H(g)^{\prime}$. Therefore, the assertion (i) is a direct consequence of Theorem 3.10(i).

(ii) Let $(x, u) \in C([0, T] ; E) \times C([0, T] ; V)$ be the unique solution to differential variational-hemivariational inequality (1). We now discuss the following time-dependent variational-hemivariational inequality: find $\tilde{u}_{\rho} \in$ $C([0, T] ; V)$ such that

$$
\begin{gathered}
\left\langle g\left(t, x(t), \tilde{u}_{\rho}(t)\right), v-\tilde{u}_{\rho}(t)\right\rangle+\frac{1}{\rho}\left\langle P \tilde{u}_{\rho}(t), v-\tilde{u}_{\rho}(t)\right\rangle \\
+J^{0}\left(\gamma \tilde{u}_{\rho}(t) ; \gamma\left(v-\tilde{u}_{\rho}(t)\right)\right)+\varphi\left(v, \tilde{u}_{\rho}(t)\right) \geq 0
\end{gathered}
$$

for all $v \in V$ and all $t \in[0, T]$. From Theorems 3.6 and 3.10, it is obvious that problem (22) admits a unique solution $\tilde{u}_{\rho} \in C([0, T] ; V)$.

Let $u_{0} \in K$ be fixed. Inserting $v=u_{0}$ into (22), it yields

$$
\begin{gathered}
\left\langle g\left(t, x(t), \tilde{u}_{\rho}(t)\right), u_{0}-\tilde{u}_{\rho}(t)\right\rangle+\frac{1}{\rho}\left\langle P \tilde{u}_{\rho}(t), u_{0}-\tilde{u}_{\rho}(t)\right\rangle \\
+J^{0}\left(\gamma \tilde{u}_{\rho}(t) ; \gamma\left(u_{0}-\tilde{u}_{\rho}(t)\right)\right)+\varphi\left(u_{0}, \tilde{u}_{\rho}(t)\right) \geq 0
\end{gathered}
$$

for all $t \in[0, T]$. The monotonicity of $g$ and the above inequality infer

$$
\begin{aligned}
m_{g} & \left\|u_{0}-\tilde{u}_{\rho}(t)\right\|_{V}^{2} \\
\leq & \left\langle g\left(t, x(t), \tilde{u}_{\rho}(t)\right)-g\left(t, x(t), u_{0}\right), \tilde{u}_{\rho}(t)-u_{0}\right\rangle \\
\leq & \frac{1}{\rho}\left\langle P \tilde{u}_{\rho}(t), u_{0}-\tilde{u}_{\rho}(t)\right\rangle+J^{0}\left(\gamma \tilde{u}_{\rho}(t) ; \gamma\left(u_{0}-\tilde{u}_{\rho}(t)\right)\right) \\
\quad & +\varphi\left(u_{0}, \tilde{u}_{\rho}(t)\right)+\left\langle g\left(t, x(t), u_{0}\right), u_{0}-\tilde{u}_{\rho}(t)\right\rangle
\end{aligned}
$$


for all $t \in[0, T]$. Notice the facts that $P v=0$ for all $v \in K, P$ is monotone and $u_{0} \in K$, it reads

$$
\begin{aligned}
m_{g} \| & u_{0}-\tilde{u}_{\rho}(t) \|_{V}^{2} \\
\leq & \frac{1}{\rho}\left\langle P \tilde{u}_{\rho}(t), u_{0}-\tilde{u}_{\rho}(t)\right\rangle \\
& +J^{0}\left(\gamma \tilde{u}_{\rho}(t) ; \gamma\left(u_{0}-\tilde{u}_{\rho}(t)\right)\right) \\
& +\varphi\left(u_{0}, \tilde{u}_{\rho}(t)\right)+\left\langle g\left(t, x(t), u_{0}\right), u_{0}-\tilde{u}_{\rho}(t)\right\rangle \\
= & \frac{1}{\rho}\left\langle P \tilde{u}_{\rho}(t)-P u_{0}, u_{0}-\tilde{u}_{\rho}(t)\right\rangle+J^{0}\left(\gamma \tilde{u}_{\rho}(t) ; \gamma\left(u_{0}-\tilde{u}_{\rho}(t)\right)\right) \\
& +\varphi\left(u_{0}, \tilde{u}_{\rho}(t)\right)+\left\langle g\left(t, x(t), u_{0}\right), u_{0}-\tilde{u}_{\rho}(t)\right\rangle \\
\leq & J^{0}\left(\gamma \tilde{u}_{\rho}(t) ; \gamma\left(u_{0}-\tilde{u}_{\rho}(t)\right)\right)+\varphi\left(u_{0}, \tilde{u}_{\rho}(t)\right)+\left\langle g\left(t, x(t), u_{0}\right), u_{0}-\tilde{u}_{\rho}(t)\right\rangle
\end{aligned}
$$

for all $t \in[0, T]$. Recall that $v \mapsto \varphi\left(u_{0}, v\right)$ is concave and upper semicontinuous. This means that $v \mapsto-\varphi\left(u_{0}, v\right)$ is convex and lower semicontinuous. The latter coupled with [33, Proposition 1.29] infers that there exist an element $\eta_{\varphi} \in V^{*}$ and a constant $c_{\varphi} \in \mathbb{R}$, which just depend on $u_{0}$, fulfilling

$$
-\varphi\left(u_{0}, v\right) \geq\left\langle\eta_{\varphi}, v\right\rangle+c_{\varphi} \text { for all } v \in V
$$

Applying hypothesis $H(J)^{\prime}(\mathrm{i})$ and $H(\varphi)(\mathrm{iii})$, we are able to find

$$
\begin{aligned}
\left(m_{g}\right. & \left.-m_{J}\|\gamma\|_{\mathcal{L}(V, X)}^{2}\right)\left\|u_{0}-\tilde{u}_{\rho}(t)\right\|_{V}^{2} \\
\leq & m_{g}\left\|u_{0}-\tilde{u}_{\rho}(t)\right\|_{V}^{2} \\
& -\left(J^{0}\left(\gamma \tilde{u}_{\rho}(t) ; \gamma\left(u_{0}-\tilde{u}_{\rho}(t)\right)\right)+J^{0}\left(\gamma u_{0} ; \gamma\left(\tilde{u}_{\rho}(t)-u_{0}\right)\right)\right) \\
\leq & -J^{0}\left(\gamma u_{0} ; \gamma\left(\tilde{u}_{\rho}(t)-u_{0}\right)\right)+\varphi\left(u_{0}, \tilde{u}_{\rho}(t)\right) \\
& +\left\langle g\left(t, x(t), u_{0}\right), u_{0}-\tilde{u}_{\rho}(t)\right\rangle \\
\leq & -\left\langle\gamma^{*} \xi_{u_{0}}, \tilde{u}_{\rho}(t)-u_{0}\right\rangle+\varphi\left(u_{0}, \tilde{u}_{\rho}(t)\right) \\
& +\left\langle g\left(t, x(t), u_{0}\right), u_{0}-\tilde{u}_{\rho}(t)\right\rangle \\
\leq & \left(\left\|g\left(t, x(t), u_{0}\right)\right\|_{V^{*}}+\left\|\gamma^{*} \xi_{u_{0}}\right\|_{V^{*}}\right)\left\|\tilde{u}_{\rho}(t)-u_{0}\right\|_{V} \\
& +\left\|\eta_{\varphi}\right\|_{V^{*}}\left\|\tilde{u}_{\rho}(t)\right\|_{V}+\left|c_{\varphi}\right| \\
\leq & \left(\left\|g\left(t, x(t), u_{0}\right)\right\|_{V^{*}}+\|\gamma\|_{\mathcal{L}(V, X)}\left(\alpha{ }_{J}+b_{J}\left\|\gamma u_{0}\right\|_{X}\right)\right)\left\|\tilde{u}_{\rho}(t)-u_{0}\right\|_{V} \\
& +\left\|\eta_{\varphi}\right\|_{V^{*}}\left\|\tilde{u}_{\rho}(t)\right\|_{V}+\left|c_{\varphi}\right| \\
\leq & \left(\left\|g\left(t, x(t), u_{0}\right)\right\|_{V^{*}}+\|\gamma\|_{\mathcal{L}(V, X)}\left(\alpha{ }_{J}+b_{J}\left\|\gamma u_{0}\right\|_{X}\right)\right. \\
& \left.+\left\|\eta_{\varphi}\right\|_{V^{*}}\right)\left\|\tilde{u}_{\rho}(t)-u_{0}\right\|_{V} \\
& +\left\|\eta_{\varphi}\right\|_{V^{*}}\left\|u_{0}\right\|_{V}+\left|c_{\varphi}\right|
\end{aligned}
$$

for all $t \in[0, T]$, where $\xi_{u_{0}} \in X^{*}$ is such that

$$
J^{0}\left(\gamma u_{0} ; \gamma\left(\tilde{u}_{\rho}(t)-u_{0}\right)\right)=\left\langle\xi_{u_{0}}, \gamma\left(\tilde{u}_{\rho}(t)-u_{0}\right)\right\rangle_{X^{*} \times X}
$$


However, hypothesis $H(g)^{\prime}(\mathrm{ii})$ points out

$$
\begin{aligned}
& \left\|g\left(t, x(t), u_{0}\right)\right\|_{V^{*}} \\
& \quad \leq\left\|g\left(t, x(t), u_{0}\right)-g\left(0,0_{E}, u_{0}\right)\right\|_{V^{*}}+\left\|g\left(0,0_{E}, u_{0}\right)\right\|_{V^{*}} \\
& \quad \leq L_{g}\left(|t|+\|x(t)\|_{E}\right)+\left\|g\left(0,0_{E}, u_{0}\right)\right\|_{V^{*}} \\
& \quad \leq L_{g}\left(T+\|x\|_{C([0, T] ; E)}\right)+\left\|g\left(0,0_{E}, u_{0}\right)\right\|_{V^{*}} .
\end{aligned}
$$

Combining (23) with (24), we have

$$
\left(m_{g}-m_{J}\|\gamma\|_{\mathcal{L}(V, X)}^{2}\right)\left\|u_{0}-\tilde{u}_{\rho}(t)\right\|_{V}^{2} \leq c_{1}\left\|\tilde{u}_{\rho}(t)-u_{0}\right\|_{V}+c_{2},
$$

where $c_{1}$ and $c_{2}$ are defined by

$$
\begin{aligned}
c_{1}:= & L_{g}\left(T+\|x\|_{C([0, T] ; E)}\right)+\|\gamma\|_{\mathcal{L}(V, X)}\left(\alpha_{J}+b_{J}\left\|\gamma u_{0}\right\|_{X}\right) \\
& +\left\|\eta_{\varphi}\right\|_{V^{*}}+\left\|g\left(0,0_{E}, u_{0}\right)\right\|_{V^{*}}, \\
c_{2}:= & \left\|\eta_{\varphi}\right\|_{V^{*}}\left\|u_{0}\right\|_{V}+\left|c_{\varphi}\right| .
\end{aligned}
$$

Further, invoking the Cauchy inequality, it finds

$$
\frac{\left(m_{g}-m_{J}\|\gamma\|_{\mathcal{L}(V, X)}^{2}\right)}{2}\left\|u_{0}-\tilde{u}_{\rho}(t)\right\|_{V}^{2} \leq \frac{c_{1}^{2}}{2\left(m_{g}-m_{J}\|\gamma\|_{\mathcal{L}(V, X)}^{2}\right)}+c_{2} .
$$

This reveals that the sequence $\left\{\tilde{u}_{\rho}(t)\right\}_{\rho>0, t \in[0, T]}$ is uniformly bounded.

Therefore, for each $t \in[0, T]$ fixed, passing to a subsequence if necessary, we may assume that

$$
\tilde{u}_{\rho}(t) \rightarrow \tilde{u}(t) \quad \text { in } V \text { as } \rho \rightarrow 0,
$$

for some $\tilde{u}(t) \in V$. We shall demonstrate that $\tilde{u}(t) \in K$. Indeed, the monotonicity of $g$ guarantees

$$
\begin{aligned}
\frac{1}{\rho}\langle & \left.P \tilde{u}_{\rho}(t), \tilde{u}_{\rho}(t)-v\right\rangle \\
\leq & \left\langle g\left(t, x(t), \tilde{u}_{\rho}(t)\right), v-\tilde{u}_{\rho}(t)\right\rangle \\
& \quad+J^{0}\left(\gamma \tilde{u}_{\rho}(t) ; \gamma\left(v-\tilde{u}_{\rho}(t)\right)\right)+\varphi\left(v, \tilde{u}_{\rho}(t)\right) \\
\leq & \left\langle g(t, x(t), v), v-\tilde{u}_{\rho}(t)\right\rangle \\
& +J^{0}\left(\gamma \tilde{u}_{\rho}(t) ; \gamma\left(v-\tilde{u}_{\rho}(t)\right)\right)+\varphi\left(v, \tilde{u}_{\rho}(t)\right)
\end{aligned}
$$

for all $v \in V$. Putting $v=\tilde{u}(t)$ into the above inequality, it has

$$
\begin{aligned}
& \frac{1}{\rho}\left\langle P \tilde{u}_{\rho}(t), \tilde{u}_{\rho}(t)-\tilde{u}(t)\right\rangle \\
& \quad \leq\left\langle g(t, x(t), \tilde{u}(t)), \tilde{u}(t)-\tilde{u}_{\rho}(t)\right\rangle+J^{0}\left(\gamma \tilde{u}_{\rho}(t) ; \gamma\left(\tilde{u}(t)-\tilde{u}_{\rho}(t)\right)\right)+\varphi\left(\tilde{u}(t), \tilde{u}_{\rho}(t)\right) .
\end{aligned}
$$

Combining with the compactness of $\gamma$ and hypotheses $H(J)^{\prime}, H(\varphi)$ (ii)-(iii), it has

$$
\limsup _{\rho \rightarrow 0}\left\langle P \tilde{u}_{\rho}(t), \tilde{u}_{\rho}(t)-\tilde{u}(t)\right\rangle \leq 0
$$


Recall that $P$ is bounded, monotone and hemicontinuous, so, it is pseudomonotone (see [25, Theorem 3.74]), which coupled with (25) and (26) implies

$$
\begin{aligned}
& \langle P \tilde{u}(t), \tilde{u}(t)-v\rangle \\
& \quad \leq \liminf _{\rho \rightarrow 0}\left\langle P \tilde{u}_{\rho}(t), \tilde{u}_{\rho}(t)-v\right\rangle \\
& \quad \leq \limsup _{\rho \rightarrow 0}\left\langle P \tilde{u}_{\rho}(t), \tilde{u}_{\rho}(t)-v\right\rangle \\
& \quad \leq 0
\end{aligned}
$$

for all $v \in V$. Since $v \in V$ is arbitrary, this indicates $P \tilde{u}(t)=0$, so we have $\tilde{u}(t) \in K$.

Additionally, we will prove $\tilde{u}(t)=u(t)$ for all $t \in[0, T]$. Now, we use the monotonicity of $g$ and $P$ as well as the fact $P v=0$ for all $v \in K$ to conclude

$$
\begin{aligned}
& \left\langle g(t, x(t), v), \tilde{u}_{\rho}(t)-v\right\rangle \\
& \leq \quad\left\langle g\left(t, x(t), \tilde{u}_{\rho}(t)\right), \tilde{u}_{\rho}(t)-v\right\rangle \\
& \leq-\frac{1}{\rho}\left\langle P v-P \tilde{u}_{\rho}(t), v\right. \\
& \left.\quad-\tilde{u}_{\rho}(t)\right\rangle+J^{0}\left(\gamma \tilde{u}_{\rho}(t) ; \gamma\left(v-\tilde{u}_{\rho}(t)\right)\right)+\varphi\left(v, \tilde{u}_{\rho}(t)\right) \\
& \quad \leq J^{0}\left(\gamma \tilde{u}_{\rho}(t) ; \gamma\left(v-\tilde{u}_{\rho}(t)\right)\right)+\varphi\left(v, \tilde{u}_{\rho}(t)\right)
\end{aligned}
$$

for all $v \in K$. Passing to the upper limit as $\rho \rightarrow 0$ in the above inequality, it has

$$
\langle g(t, x(t), v), v-\tilde{u}(t)\rangle+J^{0}(\gamma \tilde{u}(t) ; \gamma(v-\tilde{u}(t)))+\varphi(v, \tilde{u}(t)) \geq 0
$$

for all $v \in K$. Nonetheless, utilizing Minty's approach (see Lemma 3.5 as well), one has

$$
\langle g(t, x(t), \tilde{u}(t)), v-\tilde{u}(t)\rangle+J^{0}(\gamma \tilde{u}(t) ; \gamma(v-\tilde{u}(t)))+\varphi(v, \tilde{u}(t)) \geq 0
$$

for all $v \in K$. Keeping in mind that $u(t)$ is the unique solution of the inequality (2), so, we conclude that $\tilde{u}(t)=u(t)$ for all $t \in[0, T]$.

Because $\left\{\tilde{u}_{\rho}(t)\right\}$ is bounded and for each weakly convergent subsequence of $\left\{\tilde{u}_{\rho}(t)\right\}$ converges to the same limit $u(t)$, we now use [33, Theorem 1.20] to obtain that for each $t \in[0, T]$ the whole sequence $\left\{\tilde{u}_{\rho}(t)\right\}$ converges weakly to $u(t)$. On the other side, taking $v=u(t)$ in (27) and passing to the limit as $\rho \rightarrow 0$ in the resulting inequality, it suggests

$$
\lim _{\rho \rightarrow 0}\left\langle g\left(t, x(t), \tilde{u}_{\rho}(t)\right), \tilde{u}_{\rho}(t)-u(t)\right\rangle=0,
$$

here we have used the compactness of $\gamma$ and hypothesis $H(\varphi)$ (iii). Taking account of the convergence $\tilde{u}_{\rho}(t) \rightarrow u(t)$ in $V$ as $\rho \rightarrow 0$ and the monotonicity of $g$, we get

$$
\begin{aligned}
& \lim _{\rho \rightarrow 0} m_{g}\left\|u(t)-\tilde{u}_{\rho}(t)\right\|_{V}^{2} \\
& \quad \leq \lim _{\rho \rightarrow 0}\left\langle g(t, x(t), u(t))-g\left(t, x(t), \tilde{u}_{\rho}(t)\right), u(t)-\tilde{u}_{\rho}(t)\right\rangle \\
& \quad=\lim _{\rho \rightarrow 0}\left\langle g(t, x(t), u(t)), u(t)-\tilde{u}_{\rho}(t)\right\rangle-\lim _{\rho \rightarrow 0}\left\langle g\left(t, x(t), \tilde{u}_{\rho}(t)\right), u(t)-\tilde{u}_{\rho}(t)\right\rangle \\
& \quad=0
\end{aligned}
$$


for all $t \in[0, T]$. Consequently, we are able to conclude

$$
\tilde{u}_{\rho}(t) \rightarrow u(t) \text { in } V \text { as } \rho \rightarrow 0
$$

for each $t \in[0, T]$.

Assume that $\left(x_{\rho}, u_{\rho}\right) \in C([0, T] ; E) \times C([0, T] ; V)$ is the unique solution to problem (20). Then we have

$$
\begin{aligned}
& \left\langle g\left(t, x_{\rho}(t), u_{\rho}(t)\right), v-u_{\rho}(t)\right\rangle+\frac{1}{\rho}\left\langle P u_{\rho}(t), v-u_{\rho}(t)\right\rangle \\
& +J^{0}\left(\gamma u_{\rho}(t) ; \gamma\left(v-u_{\rho}(t)\right)\right)+\varphi\left(v, u_{\rho}(t)\right) \geq 0
\end{aligned}
$$

for all $v \in V$ and all $t \in[0, T]$. Inserting $v=\tilde{u}_{\rho}(t)$ and $v=u_{\rho}(t)$ into (29) and (22), respectively, we sum the resulting inequalities and apply the monotonicity of $P$ and the fact, $\varphi(v, u)+\varphi(u, v) \leq 0$ for all $u, v \in K$, to deliver

$$
\begin{aligned}
& \left\langle g\left(t, x_{\rho}(t), u_{\rho}(t)\right)-g\left(t, x(t), \tilde{u}_{\rho}(t)\right), u_{\rho}(t)-\tilde{u}_{\rho}(t)\right\rangle-J^{0}\left(\gamma u_{\rho}(t) ; \gamma\left(\tilde{u}_{\rho}(t)-u_{\rho}(t)\right)\right) \\
& \quad-J^{0}\left(\gamma \tilde{u}_{\rho}(t) ; \gamma\left(u_{\rho}(t)-\tilde{u}_{\rho}(t)\right)\right) \leq 0
\end{aligned}
$$

for all $t \in[0, T]$. The latter and conditions $H(g)^{\prime}$ hint

$$
\begin{aligned}
& \left(m_{g}-m_{J}\|\gamma\|_{\mathcal{L}(V ; X)}^{2}\right)\left\|u_{\rho}(t)-\tilde{u}_{\rho}(t)\right\|_{V}^{2} \\
& \left.\leq \leq g\left(t, x_{\rho}(t), u_{\rho}(t)\right)-g\left(t, x_{\rho}(t), \tilde{u}_{\rho}(t)\right), u_{\rho}(t)-\tilde{u}_{\rho}(t)\right\rangle \\
& \quad-J^{0}\left(\gamma u_{\rho}(t) ; \gamma\left(\tilde{u}_{\rho}(t)-u_{\rho}(t)\right)\right)-J^{0}\left(\gamma \tilde{u}_{\rho}(t) ; \gamma\left(u_{\rho}(t)-\tilde{u}_{\rho}(t)\right)\right) \\
& \leq\left\langle g\left(t, x(t), \tilde{u}_{\rho}(t)\right)-g\left(t, x_{\rho}(t), \tilde{u}_{\rho}(t)\right), u_{\rho}(t)-\tilde{u}_{\rho}(t)\right\rangle \\
& \quad \leq\left\|g\left(t, x(t), \tilde{u}_{\rho}(t)\right)-g\left(t, x_{\rho}(t), \tilde{u}_{\rho}(t)\right)\right\|_{V^{*}}\left\|u_{\rho}(t)-\tilde{u}_{\rho}(t)\right\|_{V} \\
& \quad \leq L_{g}\left\|x(t)-x_{\rho}(t)\right\|_{E}\left\|u_{\rho}(t)-\tilde{u}_{\rho}(t)\right\|_{V}
\end{aligned}
$$

for all $t \in[0, T]$. This coupled with the fact,

$$
\left\|u_{\rho}(t)-u(t)\right\|_{V} \leq\left\|u_{\rho}(t)-\tilde{u}_{\rho}(t)\right\|_{V}+\left\|\tilde{u}_{\rho}(t)-u(t)\right\|_{V}
$$

implies

$$
\left\|u_{\rho}(t)-u(t)\right\|_{V} \leq\left\|\tilde{u}_{\rho}(t)-u(t)\right\|_{V}+\frac{L_{g}}{m_{g}-m_{J}\|\gamma\|_{\mathcal{L}(V, X)}^{2}}\left\|x(t)-x_{\rho}(t)\right\|_{E}
$$

for all $t \in[0, T]$. As before we have done in the proof of Theorem 3.10, a simple calculation gives

$$
\left\|x(t)-x_{\rho}(t)\right\|_{E} \leq M_{A} \int_{0}^{t} \psi(s)\left(\left\|x(s)-x_{\rho}(s)\right\|_{E}+\left\|u_{\rho}(s)-u(s)\right\|_{V}\right) \mathrm{d} s
$$


for all $t \in[0, T]$. Combining (30) with (31) yields

$$
\begin{aligned}
& \left\|x(t)-x_{\rho}(t)\right\|_{E} \\
& \leq M_{A} \int_{0}^{t} \psi(s)\left\|\tilde{u}_{\rho}(s)-u(s)\right\|_{V} \mathrm{~d} s \\
& \quad+M_{A} \int_{0}^{t} \psi(s)\left(1+\frac{L_{g}}{m_{g}-m_{J}\|\gamma\|_{\mathcal{L}(V, X)}^{2}}\right)\left\|x(s)-x_{\rho}(s)\right\|_{E} \mathrm{~d} s
\end{aligned}
$$

for all $t \in[0, T]$. We are now in a position to employ Gronwall's inequality to find a constant $C_{0}>0$, which is independent of $\rho$, such that

$$
\left\|x(t)-x_{\rho}(t)\right\|_{E} \leq C_{0} \int_{0}^{t} \psi(s)\left\|\tilde{u}_{\rho}(s)-u(s)\right\|_{V} \mathrm{~d} s
$$

for all $t \in[0, T]$. This, together with convergence (28) and the Lebesgue dominated convergence theorem, implies

$$
\left\|x(t)-x_{\rho}(t)\right\|_{E} \rightarrow 0, \text { as } \rho \rightarrow 0
$$

for all $t \in[0, T]$. Finally, taking account of (30) and (32), we conclude the desired result (21), which completes the proof.

Finally, we provide an example for function $\varphi$.

Example 4.2. Let $\Omega$ be a bounded domain in $\mathbb{R}^{n}$. Let $V=L^{2}(\Omega)$ and $K:=$ $\{u \in V \mid u(x) \geq 0$ for a.e. $x \in \Omega\}$. Then the function $\varphi: K \times K \rightarrow \mathbb{R}$ defined by

$$
\varphi(v, u)=\int_{\Omega} v(x) u(x) \mathrm{d} x-\|u\|_{L^{2}(\Omega)}^{2}
$$

satisfies assumptions $H(\varphi)$ with $\varphi(v, u)+\varphi(u, v) \leq 0$ for all $u, v \in K$.

\section{Conclusion}

In this paper, we consider a complicated dynamic system called differential variational-hemivariational inequality in Banach spaces which consists of a time-dependent generalized variational-hemivariational inequality combined with a nonlinear evolution equation. Under quite general assumptions on the data, first, we proved a useful existence theorem, Theorem 3.8, which extends the recent result [18, Theorem 4.4]. Then we employed a fixed point theorem of history-dependent operators to show the uniqueness and stability of the solution to problem (1). Finally, the penalty methods were applied to problem (1) and a convergence result, the solution of original differential variationalhemivariational inequality (1) can be approximated by the penalized problem (20), was obtained. In the future, we plan to apply the theoretical results established in the current paper to viscoelastic contact problems with damage, and reaction-diffusion systems described by time-dependent nonsmooth semipermeability problems. 
Open Access. This article is licensed under a Creative Commons Attribution 4.0 International License, which permits use, sharing, adaptation, distribution and reproduction in any medium or format, as long as you give appropriate credit to the original author(s) and the source, provide a link to the Creative Commons licence, and indicate if changes were made. The images or other third party material in this article are included in the article's Creative Commons licence, unless indicated otherwise in a credit line to the material. If material is not included in the article's Creative Commons licence and your intended use is not permitted by statutory regulation or exceeds the permitted use, you will need to obtain permission directly from the copyright holder. To view a copy of this licence, visit http:// creativecommons.org/licenses/by/4.0/.

Publisher's Note Springer Nature remains neutral with regard to jurisdictional claims in published maps and institutional affiliations.

\section{References}

[1] Chen, X., Wang, Z.: Differential variational inequality approach to dynamic games with shared constraints. Math. Program. 146, 379-408 (2014)

[2] Chen, X., Wang, Z.: Convergence of regularized time-stepping methods for differential variational inequalities. SIAM J. Optim. 23, 1647-1671 (2013)

[3] Denkowski, Z., Migórski, S., Papageorgiou, N.S.: An Introduction to Nonlinear Analysis: Theory. Kluwer Academic/Plenum Publishers, Boston (2003)

[4] Denkowski, Z., Migórski, S., Papageorgiou, N.S.: An Introduction to Nonlinear Analysis: Applications. Kluwer Academic/Plenum Publishers, Boston (2003)

[5] Fan, K.: Some properties of convex sets related to fixed point theorems. Math. Ann. 266, 519-537 (1984)

[6] Gwinner, J.: On a new class of differential variational inequalities and a stability result. Math. Program. 139, 205-221 (2013)

[7] Han, W., Migórski, S., Sofonea, M.: On penalty method for unilateral contact problem with non-monotone contact condition. J. Comput. Appl. Math. 356, 293-301 (2019)

[8] Ke, T.D., Loi, N.V., Obukhovskii, V.: Decay solutions for a class of fractional differential variational inequalities. Fract. Calc. Appl. Anal. 18, 531-553 (2015)

[9] Li, X.J., Yong, J.M.: Optimal Control Theory for Infinite Dimensional Systems. Springer, Boston (2012)

[10] Li, X.S., Huang, N.J., O'Regan, D.: Differential mixed variational inequalities in finite dimensional spaces. Nonlinear Anal. TMA 72, 3875-3886 (2010)

[11] Li, X.S., Huang, N.J., O'Regan, D.: A class of impulsive differential variational inequalities in finite dimensional spaces. J. Franklin Inst. 353, 3151-3175 (2016)

[12] Lions, J.L., Stampacchia, G.: Variational inequalities. Commun. Pure Appl. Math. 20, 493-519 (1967)

[13] Liu, Z.H., Motreanu, D., Zeng, S.D.: Nonlinear evolutionary systems driven by mixed variational inequalities and its applications. Nonlinear Anal. RWA 42, 409-421 (2018)

[14] Liu, Z.H., Loi, N.V., Obukhovskii, V.: Existence and global bifurcation of periodic solutions to a class of differential variational inequalities. Int. J. Bifurcat. Chaos 23, ID 1350125 (2013) 
[15] Liu, Z.H., Zeng, S.D.: Differential variational inequalities in infinite Banach spaces. Acta Math. Sci. 37, 26-32 (2017)

[16] Liu, Z.H., Migórski, S., Zeng, S.D.: Partial differential variational inequalities involving nonlocal boundary conditions in Banach spaces. J. Differ. Equ. 263, 3989-4006 (2017)

[17] Liu, Z.H., Motreanu, D., Zeng, S.D.: On the well-posedness of differential mixed quasi-variational inequalities. Topol. Methods Nonlinear Anal. 51, 135-150 (2018)

[18] Liu, Z.H., Zeng, S.D., Motreanu, D.: Evolutionary problems driven by variational inequalities. J. Differ. Equ. 260, 6787-6799 (2016)

[19] Liu, Z.H., Zeng, S.D., Motreanu, D.: Partial differential hemivariational inequalities. Adv. Nonlinear Anal. 7, 571-586 (2018)

[20] Loi, N.V.: On two-parameter global bifurcation of periodic solutions to a class of differential variational inequalities. Nonlinear Anal. TMA 122, 83-99 (2015)

[21] Migórski, S., Zeng, S.D.: A class of generalized evolutionary problems driven by variational inequalities and fractional operators. Set-Valued Var. Anal. 27, 949-970 (2019)

[22] Migórski, S., Zeng, S.D.: A class of differential hemivariational inequalities in Banach spaces. J. Global Optim. 72, 761-779 (2018)

[23] Migórski, S., Zeng, S.D.: Hyperbolic hemivariational inequalities controlled by evolution equations with application to adhesive contact model. Nonlinear Anal. RWA 43, 121-143 (2018)

[24] Migórski, S., Zeng, S.D.: Mixed variational inequalities driven by fractional evolutionary equations. Acta Math. Sci. 39, 461-468 (2019)

[25] Migórski, S., Ochal, A., Sofonea, M.: Nonlinear Inclusions and Hemivariational Inequalities. Models and Analysis of Contact Problems, Advances in Mechanics and Mathematics, vol. 26. Springer, New York (2013)

[26] Migórski, S., Zeng, S.D.: Penalty and regularization method for variationalhemivariational inequalities with application to frictional contact. ZAMM Z. Angew. Math. Mech. 98, 1503-1520 (2018)

[27] Migórski, S., Ochal, A., Sofonea, M.: A class of variational-hemivariational inequalities in reflexive Banach spaces. J. Elast. 127, 151-178 (2017)

[28] Nguyen, T.V.A., Tran, D.K.: On the differential variational inequalities of parabolic-elliptic type. Math. Methods Appl. Sci. 40, 4683-4695 (2017)

[29] Pang, J.S., Stewart, D.E.: Differential variational inequalities. Math. Program. 113, 345-424 (2008)

[30] Papageorgiou, N.S., Kyritsi-Yiallourou, S.T.: Handbook of Applied Analysis. Springer, New York (2009)

[31] Pascali, D., Sburlan, S.: Nonlinear Mappings of Monotone Type. Sijthoff and Noordhoff, Alpen aan den Rijn (1978)

[32] Sofonea, M., Migórski, S.: Variational-Hemivariational Inequalities with Applications, Monographs and Research Notes in Mathematics. Chapman \& Hall/CRC, Boca Raton (2018)

[33] Sofonea, M., Matei, A.: Mathematical Models in Contact Mechanics. London Mathematical Society Lecture Notes. Cambridge University Press, Cambridge (2012) 
[34] Van, N.T., Ke, T.D.: Asymptotic behavior of solutions to a class of differential variational inequalities. Ann. Polon. Math. 114, 147-164 (2015)

[35] Wang, X., Huang, N.J.: A class of differential vector variational inequalities in finite dimensional spaces. J. Optim. Theory Appl. 162, 633-648 (2014)

[36] Zeidler, E.: Nonlinear Functional Analysis and Applications II A/B. Springer, New York (1990)

[37] Zeng, S.D., Liu, Z.H., Migórski, S.: A class of fractional differential hemivariational inequalities with application to contact problem. Z. Angew. Math. Phys. 69, 23 (2018). https://doi.org/10.1007/s00033-018-0929-6

Guo-ji Tang

College of Science

Guangxi University for Nationalities

Nanning 530006 Guangxi

People's Republic of China

e-mail: guojvtang@126.com

Jinxia Cen

College of Science

Guangxi University for Nationalities

Nanning 530006 Guangxi

People's Republic of China

e-mail: jinxiacen@163.com

Van Thien Nguyen

Department of Mathematics

FPT University

Education Zone, Hoa Lac High Tech Park, Km 29 Thang Long Highway, Thach That Ward

Hanoi

Vietnam

e-mail: Thiennv15@fe.edu.vn

Shengda Zeng

Guangxi Colleges and Universities Key Laboratory of Complex System Optimization and Big Data Processing

Yulin Normal University

Yulin 537000 Guangxi

People's Republic of China

and

Faculty of Mathematics and Computer Science

Jagiellonian University in Krakow

ul. Lojasiewicza 6

30348 Kraków

Poland

e-mail: shengdazeng@gmail.com;

shdzeng@hotmail.com;

zengshengda@163.com 